\title{
Pricing Default Risk: The Good, The Bad, and The Anomaly
}

\author{
Sara Ferreira Filipe ${ }^{*}$, Theoharry Grammatikos ${ }^{*}$, Dimitra Michala ${ }^{*}$ \\ Luxembourg School of Finance
}

February 2014

\begin{abstract}
While empirical literature has documented a negative relation between default risk and stock returns, the theory suggests that default risk should be positively priced. We provide an explanation for this "default anomaly", by calculating monthly probabilities of default (PDs) for a large sample of firms and decomposing them into systematic and idiosyncratic components. The systematic part, measured as the PD sensitivity to aggregate default risk, is positively related to stock returns. Our results show that riskier stocks underperform because they have on average lower exposures to aggregate default risk. Moreover their idiosyncratic risk is a hedge against downside market conditions.
\end{abstract}

JEL Codes: G11, G12, G15, G33

Keywords: Default Risk, Merton model, Default Anomaly, Idiosyncratic Risk

* Luxembourg School of Finance, University of Luxembourg, 4 rue Albert Borschette, L-1246, Luxembourg. Emails: sara.ferreira@uni.lu; theoharry.grammatikos@uni.lu; dimitra.michala@uni.lu. Dimitra Michala is the corresponding author (Tel: +352 4666446805).

We would like to thank Ali NasserEddine for excellent research assistance. This research is financially supported by the European Investment Bank University Research Sponsorship Program. All errors are our own. 
Finance theory suggests that, if default risk is systematic and thus non-diversifiable, it should be positively correlated with expected stock returns in the cross-section of firms. However, a number of empirical studies have delivered contradictory findings regarding the sign and significance of this relation. In this paper, we aim to bridge the gap between these seemingly puzzlingly results, by using a novel approach to study the relation between default risk and stock returns in Europe.

Early studies show that small stocks have higher returns than big stocks (Banz, 1981, the socalled size effect) and that value stocks have higher returns than growth stocks (Fama and French, 1992, the so-called value effect). In line with theory, Chan and Chen (1991) and Fama and French (1996) suggest that size and book-to-market (BM) respectively proxy for a priced default risk factor. Validating this explanation, Vassalou and Xing (2004) and Chava and Purnanandam (2010) document a positive relation between default risk and stock returns in the US. In a recent working paper, Aretz, Florackis and Kostakis (2013) report similar findings using an international sample. On the contrary, several other studies find a negative relation between default risk and returns, the so-called "default anomaly". Examples are Dichev (1998), Griffin and Lemmon (2002), Campbell, Hilscher and Szilagyi (2008), Garlappi, Shu and Yan (2008), Avramov et al. (2009), Da and Gao (2010), Garlappi and Yan (2011), and Conrad, Kapadia, and Xing (2012) in the US, and Gao, Parsons and Shen (2013) internationally. ${ }^{1}$

\footnotetext{
${ }^{1}$ Some of the explanations offered to explain this puzzling evidence are: (i) Violations of the absolute priority rule (Garlappi, Shu and Yan, 2008; Garlappi and Yan, 2011): Higher shareholder bargaining power reduces the risk of the shareholders' residual claim, thus returns close to default; (ii) Long-run risk (Avramov, Cederburg, and Hore, 2011): Firms close to default are less exposed to long-run risk because they are not expected to live long, and hence have lower returns; (iii) Glory (Conrad, Kapadia, and Xing, 2012): Firms with high default risk are glory stocks that realize high returns in the future, so their current low returns are not a good estimate of their future returns. (iv) Psychological reasons (Gao, Parsons and Shen, 2013): Investors are overconfident for high default risk stocks, keeping their prices high and subsequently leading to sudden corrections and low returns.
} 
Both literature strands above focus on the firm's physical probability of default (PD) as a measure of default risk. In most cases, they use either market-based PDs calculated under the Merton's framework, or accounting-based PDs such as Altman's Z-score, Ohlson's O-score, and the popular measure used by Campbell, Hilscher and Szilagyi (2008). Hence, these studies implicitly assume that physical PDs are monotonically related to risk-neutral PDs and that, as physical PDs increase, so does the exposure to aggregate default risk. However, George and Hwang (2010) argue that a firm's physical PD does not necessarily reflect its systematic default risk (SDR) exposure. In a theoretical model, they show that firms with high SDR exposures choose low leverage levels, which in turn lowers their physical PDs, therefore creating a negative relation between PDs and returns. In the same spirit, Kapadia (2011) finds that firms with high physical PDs do not co-vary with aggregate distress, suggesting that the low returns of high PD stocks are not due to exposure to aggregate distress. Similarly, Avramov, Cederburg and Hore (2011) show that firms with high idiosyncratic volatility (often identified as firms with high PDs) have low SDR exposures and low returns, thus suggesting a link between idiosyncratic volatility and default anomalies. ${ }^{2}$

Following George and Hwang's (2010) and Kapadia's (2010) influential work, many recent working papers use proxies of risk-neutral PDs instead of physical PDs to measure default risk, and most document a positive relation between default risk and returns. Examples are Chan-Lau (2006), Nielsen (2013), Ozdagli (2013), and Friedwald, Wagner and Zechner (2013), who use

\footnotetext{
${ }^{2}$ Other studies that document a negative relation between idiosyncratic volatility and stock returns (the IV anomaly) include Ang et al. (2006) and Barinov (2012). Also, Lopez (2004), in an earlier study, shows that under the asymptotic single risk factor approach (ASRF) used in Basel II, as a firm's PD increases and it approaches possible default, idiosyncratic factors begin to take on a more important role relative to the common, systematic risk factor. He suggests that the reasons why firms experience rising PDs are mainly idiosyncratic and not as closely linked to the general economic environment summarized by the single, common factor.
} 
credit default swap (CDS) spreads, and Anginer and Yildizhan (2013), who use corporate bond spreads to proxy for risk-neutral PDs. The main disadvantage of these studies is that they can only calculate risk-neutral PDs for firms that have CDS or bond information available. These firms constitute around $20 \%$ of total firms and are usually the largest ones. Particularly in the case of CDS, reliable data is available only after 2004.

In this paper, we extend the above recent literature and study the relation between default risk and stock returns using a new and more comprehensive approach. First, we follow Vassalou and Xing (2004) to compute monthly physical PDs (our findings are, however, robust to different methodologies). ${ }^{3}$ We then use a simple and intuitive method to decompose the estimated physical PDs into systematic and idiosyncratic components. In particular, our measure of individual firm SDR exposures is calculated as the sensitivity of the physical PD to an aggregate measure of default risk. We refer to these sensitivities as the SDR betas. As a proxy for aggregate default risk, we use the CBOE Volatility Index (VIX). Following this approach, we are able to study the relation between returns and the two components of physical PD separately and detect where the default anomaly originates. Perhaps more importantly, we can also examine a much wider sample than the studies that use CDS or bond data, with significant implications. The inclusion of smaller firms in the sample allows us to reconcile the new findings on SDR exposures with the earlier results on size and book-to-market, thus contributing to the overall understanding of default effects.

Our sample includes more than 800,000 firm-months (more than 8,000 firms), from 22 countries in Europe, during the period 1990-2012. For all of these firms, we are able to compute physical PDs and perform the subsequent decomposition (to the best of our knowledge, this is

\footnotetext{
${ }^{3}$ Vassalou and Xing (2004) describe the advantages of the Merton model versus other traditional PD measures, such as accounting models and bond information.
} 
also the first academic study to apply the Merton model to European data). The time horizon includes the introduction of the Euro and the European sovereign crisis and excludes the years before 1990, in which the majority of existing studies focuses on. Notably, we also include micro-cap stocks, which are often neglected in previous studies, but constitute the vast majority of traded firms in European exchanges.

Our approach outlined above also builds on other results in the literature. For instance, VIX is a good proxy for aggregate default risk since it is positively correlated with credit spreads, as documented in the literature on CDS (Pan and Singleton, 2008) and corporate bonds (CollinDufresne, Goldstein, and Martin, 2001; Schaefer and Strebulaev, 2008). ${ }^{4}$ Moreover, VIX is strongly correlated with European volatility indices (correlations higher than 0.90), which are generally available only from 2000 onwards. Several studies also connect VIX with stock returns. Ang et al. (2006) calculate the sensitivity of individual returns to changes in VIX, and show that firms that perform well when VIX increases experience low average returns because they are a hedge against market downside risk. Barinov (2012) additionally shows that both firms with very negative and very positive return sensitivities to VIX changes are smaller and have higher BM ratios. ${ }^{5}$ Similarly, we measure the riskiness of a firm using the sensitivity of its physical PD to VIX; a stock with low sensitivity will therefore be a safe haven against aggregate default risk. Our main hypothesis, which we confirm empirically, is that a stock with low sensitivity (not necessarily low PD) will have lower average returns, whereas investors will require a premium for holding stocks with high exposure to aggregate default risk.

\footnotetext{
${ }^{4}$ VIX is also positively correlated with other proxies of aggregate default risk, such as the mean and median PD of all firms in our sample (correlations higher than 0.50). Our results remain robust if we use the median PD instead (as Hilscher and Wilson, 2013), but this can be a rather noisy measure.

${ }^{5}$ Bansal et al. (2013) build a theoretical model that depicts these relationships.
} 
To verify this conjecture, we first sort stocks into quintile portfolios based on their physical PDs and, in line with the literature that documents a default anomaly, we find that the difference in returns between high and low PD stocks is negative and that the returns almost monotonically decrease as the PD increases. Moreover, in accordance with George and Hwang (2010), we find that stocks in the highest PD quintile have relatively low SDR exposures, as measured by the SDR betas. We then sort stocks into quintile portfolios based on their SDR betas instead; as expected, we find a positive and significant relationship between this measure of default risk and returns. Interestingly, there are non-monotonic patterns across the SDR betas portfolios. On average, the firms in the low and high SDR beta portfolios are smaller, have higher BM, and higher physical PDs than the firms in medium SDR beta portfolios. They also have higher loadings on the market and size factors, as well as higher leverage ratios (LRs) and lower return on assets (ROA). Friewald, Wagner and Zechner (2013) document the same patterns in portfolios sorted based on credit risk premia estimated from CDS spreads. These findings are evidence that our estimates of SDR exposures convey information that is different from that incorporated in traditional risk factors and stock characteristics. Finally, we show that the SDR betas are negatively related to the idiosyncratic component (measured by the alphas of the same exposure regressions, to which we refer as IDR alphas). ${ }^{6}$ As in the case of physical PDs, sorting stocks into quintiles based on this idiosyncratic component delivers evidence of a negative return relation.

Our results therefore suggest that riskier stocks, as measured by the physical PDs, will tend to underperform because they have on average lower exposures to aggregate default risk. Their riskiness is mostly idiosyncratic and can be diversified away, thus providing an explanation for

${ }^{6}$ Similarly, Avramov et al. (2013) document a negative cross-sectional relation between exposures to systematic and firm-specific risks. 
the default anomaly typically found in the literature. Further tests with double-sorting portfolios allow us to confirm these findings, i.e. high-IDR alpha stocks are a hedge against downside market conditions. On the contrary, it is the systematic component of default risk, measured by the SDR betas, that requires a return premium.

The remainder of the study is organized as follows. Section I describes the data. Section II studies the relation between the physical PDs and stock returns. Section III first describes our method to decompose the physical PDs into systematic and idiosyncratic components, and then discusses the relation between these different components and stock returns. Section IV performs further tests and provides more evidence to our explanation of the default anomaly. Finally, Section V concludes.

\section{The Data}

Our study covers publicly listed firms from the majority of countries in Europe, during the period January 1990 - December 2012. As our main data sources, we use Thomson Reuter's Datastream for market data and Thomson Reuter's Worldscope database for the firms' accounting information.

To guarantee a certain level of market exchange activity, we include in our analysis only the 22 European countries that had established exchanges on or before 1980 (for a total of 34 exchanges). We exclude years 1980-1989 due to the limited number of companies with available data. We also follow previous studies in the field and exclude financial firms ( $\mathrm{ICB}^{7} 8000$ Financials) and firms with negative BM ratios. To reduce the influence of outliers and account

\footnotetext{
${ }^{7}$ The Industry Classification Benchmark (ICB) is an industry classification taxonomy launched by Dow Jones and FTSE in 2005.
} 
for measurement errors, we exclude firms with a market capitalization below the $1^{\text {st }}$ percentile for all observations. This essentially leaves in our sample firms with a market capitalization roughly above one million euros. Moreover we only retain firms that have at least two years of data available, so we have enough history for the calculation of physical PDs. To avoid duplicate observations, we do the following. For firms that are traded in more than one European exchange, we keep data from the market where the firm has been traded for the longest period. This is almost always the home market. Finally, if a firm has issued more than one type of common shares, we use data of the share type that constitutes the majority of common equity.

An important feature of our database is the compiled information on default events. As the reason for delisting is not usually available in Datastream, we manually track the status of the delisted firms from other sources (such as Amadeus and Orbis Europe databases), as well as various public internet sources. Therefore, we are able to identify if a firm delisting is due to default (bankruptcy or liquidation) or other reasons (i.e. mergers). To illustrate this point, Table 1 reports the average number of active firms per year, as well as the number of firms that were delisted due to default each year. Nonetheless, the information on delisting returns is also not available in Datastream. Thus we follow Campbell, Hilscher and Szilagyi (2008) and use the last available full-month return, assuming that our portfolios sell stocks that are delisted (due to default) at the end of the month before delisting. ${ }^{8}$

\section{(TABLE 1)}

After applying the filters described above and merging different data sources, we are able to calculate physical PDs and draw results for a final sample of 806,157 firm-months

\footnotetext{
${ }^{8}$ This approach gives a conservative estimate of the default anomaly. Results are qualitatively the same if we follow Vasssalou and Xing (2004) and set delisting returns for stocks that default equal to -100 percent (assuming a zero recovery rate).
} 
(corresponding to 8,439 firms) across the 22 European countries. Table 2 characterizes this final sample with respect to the distribution of firms across size classes and countries. Unlike most previous studies, we include nano and micro-cap stocks, which constitute the vast majority of traded firms in European exchanges. In terms of international breakdown, the representativeness of the different countries in our sample seems to be in line with the literature (e.g. Gao, Parsons, and Shen, 2013). Unsurprisingly, more developed markets contribute with a greater share of observations to the sample, with the U.K. (32.54\%), France (13.34\%) and Germany (13.08\%) collectively comprising more than half of it.

(TABLE 2)

We also resort to various other public data sources. Regarding volatility indexes, we use the CBOE VIX, as well as the European indices VSTOXX, VFTSE and VDAX (for EUROSTOXX 50, FTSE 100 and DAX respectively). We focus on VIX in the main analysis, as it is the only index available from January 1990 on. The Fama-French factors SMB and HML and the market factor EMKT for Europe are obtained from Kenneth French's web page. For the risk-free rate, we use monthly observations of the 1-year T-bill, available from the Federal Reserve Board Statistics. $^{9}$

\section{The Physical Probabilities of Default and Stock Returns}

\section{A. Calculating Physical PDs}

We follow Vassalou and Xing (2004) in calculating our main physical PD measure. As their methodology is based on the Merton model, we also refer to the estimated physical PD as the

\footnotetext{
${ }^{9}$ We use a US risk-free rate since we do not have long enough time series of data for the German equivalent. Similarly, Kenneth French calculates the European factors using a US risk-free rate.
} 
Merton measure. In order to calculate monthly PDs under this approach, we use data on current and long-term debt, as well as market capitalization for all the firms in our sample. ${ }^{10} \mathrm{We}$ perform all calculations for the individual monthly PDs in local currency to minimize the effect of exchange rate volatility. Appendix A presents more details on the Merton measure, its calculation and overall performance.

Table 3 shows descriptive statistics for the estimated Merton measure by country. Since other firm characteristics, such as size and BM ratios, have been associated to default risk in the literature, Table 3 also includes descriptive statistics for these variables (along with raw average returns). Overall the results show that there is significant heterogeneity among European countries, in terms of PDs, size, and BM. Markets such as Romania (16.69\%) and Bulgaria (14.29\%) have the highest average PDs, and other countries such as Switzerland (3.13\%) and the Netherlands (3.42\%) have very low average PDs.

\section{(TABLE 3)}

Although the performance results in Appendix A suggest that the Merton measure is indeed a good default predictor, we also calculate an alternative default measure for robustness purposes. In particular, we follow Campbell, Hilscher and Szilagyi (2008) in calculating a physical PD measure using a multi-period logit regression framework. We refer to this alternative PD as the CHS measure. We are able to calculate the CHS measure for 755,243 firm-months (7,980 firms). For more details on the methodology, please refer to Appendix B.

Figure 1 summarizes the results. In Panel A, we plot the monthly aggregate Merton and CHS measures for firms in the overall sample (defined as simple averages of the values of all firms).

\footnotetext{
${ }^{10}$ We obtain the firm's "Current Liabilities" (WC03101), "Long-Term Debt" (WC03251) and "Common Equity" (WC03501) from Worldscope's annual accounting data. Daily market values are from Datastream.
} 
The two PD measures have a very high correlation of 0.92 , but their magnitude is different and the CHS measure produces lower PDs than the Merton measure. The columns in the plot denote recession periods in the Euro area (as indicated by the OECD), so we can also observe that both measures vary greatly with the business cycle and increase during downturns. Panel B plots the monthly aggregate Merton measure and values of the volatility index VIX at the end of each month. It is again apparent that Merton PDs and VIX comove closely together throughout the economic cycle. Both are higher during recessions, when economic theory suggests that the stochastic discount factor is high. This finding provides initial evidence that VIX captures aggregate default risk information.

\section{(FIGURE 1)}

For brevity reasons, and given the high correlation between the two PD measures, we only use the estimated Merton measure to present the results. We justify this choice in two ways. First, the CHS measure may suffer from a look-ahead bias, since we use data from the whole sample period to estimate PDs. Second, we are able to estimate the CHS measure for a smaller sample of firms compared to the Merton case. Nonetheless, our results are robust to the choice of physical PD measure.

\section{B. The Default Anomaly: Physical PDs and Stock Returns}

As a first part of our analysis, we study the possible existence of a default anomaly in Europe. In particular, we explore the cross-sectional relation between stock returns and default risk by conducting portfolio sorts on the physical PDs, i.e. the Merton measure.

Each month, from January 1990 to December 2012, we use the most recent PD for each firm and sort the stocks into five portfolios. To account for possible country effects (concentration of 
risky stocks in certain countries and/or accounting differences), we follow an approach similar to Lewellen (1999) and Barry et al. (2002): at the beginning of each month, we adjust the available PDs from stocks in the overall sample by the average country PD. Then we sort all stocks into portfolios based on the adjusted PDs. ${ }^{11}$

Table 4 reports the results. In Panel A, we report both equally and value-weighted monthly raw and risk-adjusted returns (alphas) of the five portfolios. We also construct high-low portfolios, which go long the $20 \%$ highest PD stocks and short the 20\% lowest PD stocks, and report raw returns and alphas for these portfolios (the alphas are obtained using the factormimicking portfolios for Europe available on Kenneth French's website). The results show that the difference in returns between high and low PD stocks is almost always negative, in line with the literature that documents a possible default anomaly (i.e. a puzzling negative relation between default risk and returns). This relation is almost monotonic, but differences are not always significant. Thus, there is some evidence that the highest PD stocks earn on average lower returns than the lowest PD stocks, though this underperformance does not demonstrate strong significance.

\section{(TABLE 4)}

In Panel B of Table 4, we report the estimated factor loadings for excess equally and valueweighted returns on the four Fama-French-Carhart factors. We find that high PD portfolios have

\footnotetext{
${ }^{11}$ If the integration among European markets is high, it is not necessary to adjust the PDs by the country average. Nevertheless, our sample consists of 22 European countries and three of them are not members of the European Union, thus it is not very plausible to assume a very high degree of integration. Gao, Parsons and Shen (2013) in a recent working paper follow a different approach to neutralize country effects: at the end of each month, they sort stocks within every country based on their PD and then form pooled portfolios. This way they ensure an even representation of all countries in every portfolio. However, their strategy might lead to aggregation of stocks with very heterogeneous default characteristics in the same portfolio and attribution of stocks with very similar default characteristics to different portfolios.
} 
higher loadings on the market factor (EMKT), the size factor (SMB) and the value factor (HML). This shows the prevalence of small and value stocks in the high PD portfolios. To complement this analysis, in Panel $\mathrm{C}$ we report some relevant characteristics of the five portfolios. As shown, the variation in PD is quite high among the portfolios. Stocks in the lowest PD quintile have an average PD close to zero, whereas stocks in the highest PD quintile have a PD above $22 \%$. Average size is monotonically decreasing along the portfolios and average BM is monotonically increasing, again reflecting the dominance of small and high BM firms among the high PD stocks. Specifically, stocks in the highest PD portfolio are on average around 10 times smaller than stocks in the low PD portfolio and have BM around three times higher. The high PD stocks also have high leverage ratios (LRs) and, in accordance with Chen and Zhang (2010), low return on assets (ROA).

\section{Understanding Default Effects}

\section{A. Decomposing the Physical PDs into Systematic and Idiosyncratic Components}

\section{A.1. The Motivation}

Our findings in the previous section appear to be supportive of the existence of a default anomaly, since an investing strategy that buys the highest PD stocks and shorts the lowest PD stocks has on average negative returns. At a first glance, these results suggest that default risk is, at best, not priced in the cross-section of stock returns. However finance theory suggests that, only if default risk is systematic and thus non-diversifiable, it should be positively correlated with expected stock returns. In other words, investors demand a premium to hold stocks of firms with high exposures to aggregate default risk, not necessarily firms with high physical PDs. In fact, George and Hwang (2010) argue that a firm's physical PD does not necessarily reflect its 
SDR exposure. In a theoretical model, they show that firms with high exposures to aggregate default risk choose a low leverage level, which in turn lowers their physical PDs and creates a negative relation between PDs and returns. Hence, several recent studies use limited samples where CDS or bond data is available to calculate proxies of risk-neutral PDs, and most of these studies document a positive relation between default risk and returns. Therefore we now investigate empirically if the physical PDs, calculated using the Merton approach applied to a large sample of firms, are a good measure of firm exposure to aggregate default risk.

\section{A.2. The Methodology}

To calculate SDR exposures, we follow the approach of Hilscher and Wilson (2013) and Anginer and Yildizhan (2013), by assuming that a firm's PD is exposed to a single common factor. This factor is the aggregate default risk. Therefore the firm's SDR exposure is measured as the sensitivity of its PD to this factor (we refer to this sensitivity as the SDR beta). To compute monthly SDR betas for all firms in our sample, we estimate the following regression for each firm over 24-months rolling windows:

$$
P D_{i, t}=\alpha_{i}^{I D R}+\beta_{i}^{S D R} X_{t}+\varepsilon_{i, t}
$$

where $P D_{i, t}$ is the physical PD for firm $i$ in month $t$ (i.e. the Merton measure), $X_{t}$ is the aggregate default risk measure, $\alpha_{i}^{I D R}$ is the IDR alpha and $\beta_{i}^{S D R}$ is the SDR beta for firm $i$ in month $t$, obtained from the rolling regressions method. ${ }^{12} \mathrm{We}$ are able to calculate SDR betas and

\footnotetext{
${ }^{12}$ The specification in (1) does not of itself constrain the PD to lie between zero and one. Hilscher and Wilson (2013) argue that this is not a problem, as long as most of the estimated PDs are small (so that $P(1-P) \approx P)$. Our estimated PDs satisfy this condition.
} 
IDR alphas for 624,084 firm-months (7,140 firms) for the period from January 1992 to December 2012. ${ }^{13}$

\section{A.3. VIX and Aggregate Default Risk}

As a proxy for aggregate default risk, we use the volatility index VIX. We are not the first to link VIX with default risk. Several studies find VIX to be an important determinant of credit spreads, as shown in the literature on CDS (Pan and Singleton, 2008) and on corporate bonds (CollinDufresne, Goldstein, and Martin, 2001; Schaefer and Strebulaev, 2008). Table 5 motivates further the use of VIX in our empirical analysis. Panel A presents summary statistics for VIX and its monthly change, $\Delta_{\mathrm{m}}$ VIX. Panel B reports the highly positive correlation coefficients between VIX and three European volatility indices, which suggests that VIX successfully captures aggregate volatility in Europe. Panel $\mathrm{C}$ of Table 5 reports the negative correlation coefficients between $\Delta_{\mathrm{m}}$ VIX and the monthly change of two widely used European stock indices, EUROSTOXX 50 and MSCI Europe. This finding is in line with the theoretical model of Bansal et al. (2013), according to which stock returns have on average negative volatility betas. Panel D of Table 5 reports the negative correlation coefficients of $\Delta_{\mathrm{m}} \mathrm{VIX}$ with EMKT and SMB, which is in accordance with And et al. (2006). For HML, the correlation is very low. Last, the regression results of Panel E show that VIX can explain a substantial portion of time-variation in both the aggregate and the median physical PD, as measured by the Merton measure (the results are robust if we use the CHS measure instead). ${ }^{14}$

\footnotetext{
13 The sample is smaller than before because we need two years of PD history for the estimation. Essentially, we cannot calculate SDR betas for January 1990 to December 1991.

${ }^{14}$ For robustness purposes, we follow Hilscher and Wilson (2013) and use the median PD as an alternative proxy for aggregate default risk. Hilscher and Wilson (2013) find that the median PD is highly correlated with the first principal component which explains the majority of variation in PDs across ratings. However, in our large sample of very heterogeneous countries, the median PD can be a rather
} 
(TABLE 5)

\section{A.4. Physical PDs, Systematic Betas, and Idiosyncratic Alphas}

Does the physical PD accurately reflect the firm's SDR exposure? We argue that this is not the case. In accordance with George and Hwang (2010), we find that stocks in the highest PD quintile have high leverage but relatively low SDR exposures, as measured by the SDR betas. These stocks also have very high positive IDR alphas (see Table 4, Panel C), thus a large fraction of their default risk is attributable to the idiosyncratic component. These findings provide initial evidence that the documented default anomaly may be explained by the use of physical PDs as the default measure. Therefore, we now turn to the analysis of the relations between stocks returns and the two components of default separately.

\section{B. SDR Betas and Stock Returns: A Premium on Exposures to Aggregate Default Risk}

To examine if exposures to aggregate default risk are rewarded in the cross-section of stock returns, we repeat the portfolio analysis of Section II.B now using the SDR betas as the sorting variable. Each month, from January 1992 to December 2012, we use the most recent SDR beta for each firm and sort the stocks into five portfolios. As before, we adjust monthly SDR betas by their monthly country average. Table 6 reports the results. ${ }^{15}$

\section{(TABLE 6)}

Panel A shows that the difference in returns between high and low SDR beta stocks is now always positive for both equally and value-weighted returns and significant in the case of

noisy measure. Since all our results are unchanged when we use median PD as a proxy for aggregate default risk, we only present here the results using VIX.

${ }^{15}$ As discussed above, we only report results with the VIX SDR beta as a measure of exposure to aggregate default risk, but our results are robust if we use the median SDR beta instead. 
equally-weighted returns. A portfolio strategy buying the highest SDR beta quintile and shorting the lowest SDR beta quintile of stocks gives an equally-weighted four-factor alpha of 0.33 percent monthly (4.01 percent annually), significant at a five percent level. The positive relation between returns and SDR betas is almost always monotonic. Thus, when we use an SDR measure to sort the stocks, there is evidence of a positive relation between default risk and returns, in line with theoretical models. ${ }^{16}$

In Panel B, we see that factor loadings on the market factor (EMKT) and the size factor (SMB) do not decrease monotonically along the SDR beta portfolios. Specifically, both high and low SDR beta stocks have higher loadings than medium SDR beta stocks. This indicates that small stocks are not homogeneous with respect to their SDR exposures. The factor loadings on the value factor (HML) are mostly insignificant. These results suggest that our SDR measure conveys information that is not captured by traditional risk factors.

Panel C reports some characteristics of the portfolios. First, SDR betas exhibit large crosssectional dispersion, ranging from -0.62 to 0.89 , indicating that the effect of aggregate default risk varies substantially across stocks. In accordance with Barinov (2013), negative SDR betas indicate that these portfolios are indeed a good hedge against increases in VIX, which justifies their low returns. Second, we find interesting non-monotonic patters across the beta portfolios: (a) both high and low SDR beta stocks have higher PDs than medium SDR beta stocks; (b) they also have higher LRs and lower ROA; (c) they are also, on average, smaller in size and have

\footnotetext{
${ }^{16} \mathrm{Da}$ and Gao (2010) argue that the high returns of risky stocks are not compensation for SDR, but the result of short-term return reversal caused by price pressure in the month of portfolio formation. Thus, in accordance with the default anomaly literature, they find that risky stocks deliver low returns if the second month after portfolio formation is used instead. To address this critique, we test for return persistence in our SDR beta sorted portfolios. We find no evidence of return reversal: the return of the highest and lowest SDR beta quintiles differ 8 months before portfolio formation, the difference is maximized in the portfolio formation month, and persists for almost 8 months after portfolio formation (even if we assume zero recovery in defaults).
} 
higher BM ratios (which is consistent with the results from portfolio sorts on credit risk premia estimated from CDS spreads by Friewald, Wagner and Zechner, 2013). Therefore the SDR beta conveys information that is different from that incorporated in other common default risk measures and stock characteristics. Finally, we find a negative relation between SDR betas and IDR alphas, as the idiosyncratic component of the PD increases almost monotonically across the SDR beta portfolios. This is in accordance with Avramov et al. (2013), who document a negative cross-sectional relation between exposures to systematic and firm-specific risks.

To conclude, the findings in this section show that SDR betas, measured as sensitivities of the physical PDs to a common aggregate default risk factor (here VIX) are positively related to stock returns and that high PD stocks can have quite different SDR betas among them. ${ }^{17}$

\section{IDR Alphas and Stock Returns: A negative relation}

We now sort stocks based on the IDR alphas. ${ }^{18}$ Each month, from January 1992 to December 2012, we use the most recent IDR alpha for each firm and sort the stocks into five portfolios. As before, we adjust monthly IDR alphas by their country average for this month. Table 7 reports the results.

\section{(TABLE 7)}

Panel A shows that the difference in returns between high and low IDR alpha stocks is negative for both equally and value-weighted returns, as in the case of PDs. It is also significant

\footnotetext{
${ }^{17}$ In unreported results, available upon request, we use double-sorted portfolios to analyze the relation between returns and SDR betas while controlling for the physical PD. We find that the exposure to aggregate default risk is significantly rewarded for stocks with low PDs, which are typically stocks less subject to market imperfections.

${ }^{18}$ Our results are robust if we measure the idiosyncratic component of default risk as the sum of IDR alphas and residuals from regression (1).
} 
at a five percent level for value-weighted returns and CAPM alphas. In Panel B, we see that factor loadings on the market factor (EMKT) and the size factor (SMB) do not decrease monotonically along the IDR alpha portfolios, but they follow the same patterns as for SDR beta portfolios. Specifically, both high and low IDR alpha stocks have higher loadings than medium IDR alpha stocks. As before, the factor loadings on the value factor (HML) are not significant. Panel C reports some characteristics of the portfolios. IDR alphas exhibit large cross-sectional dispersion, ranging from -8.5594 to 22.5424 . In accordance with our previous findings on SDR beta portfolios, both high and low IDR alpha stocks have higher PDs, are smaller, have higher BM and LRs, and lower ROA than medium IDR alpha stocks. As before, we document a negative relation between SDR betas and IDR alphas. Therefore, stocks that have low exposures to aggregate default risk are associated with high firm-specific risks. These results are initial evidence that the default anomaly can be explained by the non-monotonic relationship between the physical PD and its idiosyncratic component.

\section{Explaining the Default Anomaly}

This section sheds more light on the relation between default risk and stock returns. Our main focus is to understand what the main drivers of the default anomaly are, and therefore we apply a sequential two-sort procedure to investigate it. Given the results above, we sort on physical PDs while controlling for the idiosyncratic level of default risk. We use tertiles instead of quintiles to guarantee an adequate number of stocks in all portfolios. Specifically, each month, we first sort stocks into three portfolios based on their country-adjusted IDR alpha and, within each IDR alpha portfolio, we further sort stocks in three portfolios, based on the country-adjusted physical 
PD. For brevity, we report value-weighted returns but results remain qualitatively similar for equally-weighted returns. Table 8 reports the results.

\section{(TABLE 8)}

Panel A shows the time-series monthly average of the value-weighted returns and alphas, as well as average monthly returns and alphas for portfolios going long the highest PD tertile and short the lowest PD tertile of stocks. Interestingly, we find that the default anomaly is significant only for stocks in the highest IDR alpha tertile, but it is absent in the other two IDR alpha tertiles. Thus, the difference in returns between high and low PD portfolios is negative and significant only when the idiosyncratic component of the PD is very high. Panel B reports various characteristics of each portfolio. Both stocks in the highest and lowest IDR alpha tertiles have higher PDs than stocks in the medium IDR alpha tertile. Still, low IDR alpha stocks have lower PD levels than stocks in the high IDR alpha portfolio. They also differ in terms of their SDR betas. While stocks in the highest IDR alpha tertile have, on average, negative SDR betas, indicating that they are a good hedge against aggregate default risk (which explains their low returns), stocks in the lowest IDR alpha tertile have high SDR betas Another interesting finding is that, in the lowest IDR alpha tertile, as PD increases, SDR betas rise and IDR alphas fall. This shows that, for stocks with low idiosyncratic risk, the physical PD is a better proxy to exposures to aggregate default risk. Finally, size and ROA decrease and BM and LR increase monotonically as PD increases in all three IDR alpha tertiles, indicating that stocks with high PDs are, on average small, value stocks, with high leverage and low profitability.

Overall, the results above show that the negative relation between physical PD and returns is only present for stocks with very high firm-specific risk. High IDR alpha stocks have, on average, negative exposures to aggregate default risk, thus constituting a hedge against bad 
market conditions. Moreover, among high IDR alpha stocks, this hedging ability increases as PD increases (i.e. the SDR betas become more negative). We therefore argue that (1) the so-called "default anomaly" is only found in firms with high idiosyncratic risk and (2) it is not an "anomaly", in the sense that the negative returns on the High-Low PD portfolios are compensated by its hedging ability. On the contrary, for low IDR alpha stocks, the physical PD is a better measure of the firm's sensitivity to aggregate default risk; thus, in this case, higher PD is rewarded with higher returns.

\section{Conclusions}

In this paper, we shed more light on the recent contradictory literature that studies the relation between default risk and stock returns. We first follow the Merton model to calculate monthly physical probabilities of default for individual firms. We then use a novel approach to decompose these estimated PDs into systematic and idiosyncratic components. Unlike previous studies, our methodology does not require data on bonds or CDS markets. It therefore allows us to carry the analysis for a more comprehensive sample of European firms, which notably includes micro-cap firms. This heterogeneity is important as previous work has often associated default risk to other firm characteristics such as size and book-to-market ratios.

Initially, we find evidence consistent with a possible default anomaly, i.e. stocks with high physical PDs have on average lower returns. However, a closer look shows that the physical PD is usually a poor measure of exposures to aggregate default risk. Using estimated SDR betas to sort the stocks, we document a positive and significant relation between default risk and returns. In other words, investors indeed require a premium to hold stocks that are riskier when aggregate default risk is higher. Therefore it is the idiosyncratic, not the systematic part, driving the default 
anomaly. We confirm this conjecture by showing that stocks sorted on firm-specific risk have on average lower returns. Investors do not require compensation to hold stocks with high firmspecific risk because these stocks are a source of portfolio risk diversification. In fact, we show that stocks with high IDR alphas also have lower (negative) SDR betas. A double-sort test, where we sort stocks based on their physical PDs after controlling for IDR alphas, finds that the negative relation between risk and returns is significant only for high IDR alpha stocks.

Our results therefore suggest that riskier stocks, as measured by the physical PDs, will tend to underperform because they have on average lower exposures to aggregate default risk. Their riskiness is mostly idiosyncratic and can be diversified away, thus providing an explanation for the default anomaly typically found in the literature. On the contrary, it is the systematic component of default risk, measured by the SDR betas, that requires a return premium. 


\section{References}

Altman, Edward I. (1968). "Financial Ratios, Discriminant Analysis and the Prediction of Corporate Bankruptcy." The Journal of Finance 23(4), 589-609.

Ang, Andrew, Robert J. Hodrick, Yuhang Xing, and Xiaoyan Zhang. (2006). "The Cross-Section of Volatility and Expected Returns." The Journal of Finance 61(1), 259-299.

Anginer, Deniz, and Çelim Yıldızhan. (2013). "Is there a Distress Risk Anomaly? Pricing of Systematic Default Risk in the Cross Section of Equity Returns.” Working Paper.

Aretz, Kevin, Chris Florackis, and Alexandros Kostakis. (2013). "Do Stock Returns Really Decrease with Default Risk? New International Evidence.” Working paper.

Avramov, Doron, Scott Cederburg, and Satadru Hore. (2011). "Cross-Sectional Asset Pricing Puzzles: A Long-Run Perspective." Working Paper.

Avramov, Doron, Tarun Chordia, Gergana Jostova, and Alexander Philipov. (2009). "Credit Ratings and the Cross-Section of Stock Returns." Journal of Financial Markets 12(3), 469-499.

Avramov, Doron, Tarun Chordia, Gergana Jostova, and Alexander Philipov. (2013). "Anomalies and Financial Distress.” Journal of Financial Economics 108(1), 139-159.

Bansal, Ravi, Dana Kiku, Ivan Shaliastovich, and Amir Yaron. (2013). "Volatility, the Macroeconomy, and Asset Prices." The Journal of Finance, forthcoming.

Banz, Rolf W. (1981). "The Relationship between Return and Market Value of Common Stocks.” Journal of Financial Economics 9(1), 3-18.

Barinov, Alexander. (2012). "Aggregate volatility risk: Explaining the small growth anomaly and the new issues puzzle.” Journal of Corporate Finance 18(4), 763-781.

Bekaert, Geert, Campbell R. Harvey, Christian T. Ludblad, Stephan Siegel. (2013). "The European Union, the Euro, and Equity Market Integration.” Journal of Financial Economics 109(3), 583-603.

Black, Fischer, and Myron Scholes. (1973). "The Pricing of Options and Corporate Liabilities." Journal of Political Economy 81(3), 637-654.

Campbell, John Y., Jens Hilscher, and Jan Szilagyi. (2008). "In Search of Distress Risk." The Journal of Finance 63(6), 2899-2939.

Chan, L.K.C., Nai-fu Chen. (1991). "Structural and Return Characteristics of Small and Large Firms. The Journal of Finance 46(4), 1467-1484. 
Chan-Lau, J. A. (2006). Is Systematic Default Risk Priced in Equity Returns? A Cross-sectional Analysis using Credit Derivatives Prices. IMF working paper, 148.

Chava, Sudheer, Amiyatosh Purnanandam. (2010). "Is Default Risk Negatively Related to Stock Returns?" The Review of Financial Studies 23(6), 2523-2559.

Chen, Long, and Lu Zhang. (2010). "A Better Three-Factor Model That Explains More Anomalies.” The Journal of Finance 65(2), 563-594.

Collin-Dufresne, Pierre, Robert S. Goldstein, J. Spenser Martin. (2001). "The Determinants of Credit Spread Changes.” The Journal of Finance 56(6), 2177-2207.

Conrad, Jennifer, Nishad Kapadia, and Yuhang Xing. (2012) "What Explains the Distress Risk Puzzle: Death or Glory?" Working paper.

Da, Zhi, and Pengjie Gao. (2010). "Clientele Change, Liquidity Shock, and the Return on Financially Distressed Stocks.” Journal of Financial and Quantitative Analysis 45(1), 27-48.

Dichev, Ilia D. (1998). "Is the Risk of Bankruptcy a Systematic Risk?" The Journal of Finance 53(3), 1131-1147.

Fama, Eugene F., and Kenneth R. French. (1992). "The Cross-Section of Expected Stock Returns." The Journal of Finance 47(2), 427-465.

Fama, Eugene F., and Kenneth R. French. (1996). "Multifactor Explanations of Asset Pricing Anomalies." The Journal of Finance 51(1), 55-84.

Fama, Eugene F., and James D. MacBeth. (1973). "Risk, Return, and Equilibrium: Empirical Tests." Journal of Political Economy 81(3), 607-636.

Friedwald, Nils, Christian Wagner, and Josef Zechner. (2013). "Credit Risk Premia and Equity Returns." The Journal of Finance, forthcoming.

Garlappi, Lorenzo, and Hong Yan. (2011). "Financial Distress and the Cross-Section of Equity-Returns." The Journal of Finance 66(3), 789-822.

Garlappi, Lorenzo, Tao Shu, and Hong Yan. (2008). "Default Risk, Shareholder Advantage, and Stock Returns." The Review of Financial Studies 21(6), 2743-2778.

Gao, Pengjie, Christopher A. Parsons, and Jainfeng Shen. (2013). "The Global Relation Between Financial Distress and Equity Returns.” Working paper.

George Thomas J., and Chuan-Yang Hwang. (2010). "A Resolution of the Distress Risk and Leverage Puzzles in the Cross Section of Stock Returns.” Journal of Financial Economics 96(1), 56-79. 
Griffin, John M., and Michael L. Lemmon. (2002). "Book-to-Market Equity, Distress Risk and Stock Returns." The Journal of Finance 57(5), 2317-2336.

Hilscher, Jens, and Mungo Wilson. (2013). "Credit Ratings and Credit Risk: Is One Measure Enough?" Working Paper.

Kapadia, Nishad. (2011). “Tracking down Distress Risk.” Journal of Financial Economics 102(1), 167182.

Kim, Suk Joong, Fariborz Moshirian, Eliza Wu. (2006). "Evolution of International Stock and Bond Market Integration: Influence of the European Monetary Union.” Journal of Banking and Finance 30(5), 1507-1534.

Lewellen, Jonathan. (1999). "The Time-Series Relations among Expected Returns, Risk and Book-toMarket.” Journal of Financial Economics 54(1), 5-43.

Lopez, Jose A. (2004). "The Empirical Relationship between Average Asset Correlation, Firm Probability of Default, and Asset Size". Journal of Financial Intermediation 13(2), 263-283.

Merton, Robert C. (1974). "On the Pricing of Corporate Debt: The Risk Structure of Interest Rates." The Journal of Finance 29(2), 449-470.

Newey, Whitney K., and Kenneth D. West. (1987). "A Simple, Positive Semi-Definite, Heteroskedasticity and Autocorrelation Consistent Covariance Matrix.” Econometrica 55(3), 703-708.

Nielsen, Caren Yinxia G. (2013). “Is Default Risk Priced in Equity Returns?” Working Paper.

Ohlson, James A. (1980). "Financial Ratios and the Probabilistic Prediction of Bankruptcy." Journal of Accounting Research 18(1), 109-131.

Ozdagli, Ali K. (2013). "Distressed, but not risky: Reconciling the empirical relationship between financial distress, market-based risk indicators, and stock returns (and more)”. Working paper.

Pan, Jun, and Kenneth J. Singleton. (2008). "Default and Recovery Implicit in the Term Structure of Sovereign CDS Spreads.” The Journal of Finance 63(5), 2345-2384.

Schaefer, Stephen M., and Ilya A. Strebulaev. (2008). "Structural Models of Credit Risk are Useful: Evidence from Hedge Ratios on Corporate Bonds.” Journal of Financial Economics 90(1), 1-19.

Shumway, Tyler, and Vincent A. Warther. (1999). “The Delisting Bias in CRSP's Nasdaq Data and its Implications for the Size Effect.” The Journal of Finance 54(6), 2361-2379.

Vassalou, Maria, and Yuhang Xing. (2004). "Default Risk in Equity Returns." The Journal of Finance 59(2), 831-868. 
Table 1

\section{Defaulted Firms as a Percentage of Total Firms}

The table lists the total number of active firms and delistings due to default for every year of our sample period. The number of active firms is the average number of firms across all months of the year. The number of firms that were delisted due to default is hand-collected data from various public sources.

\begin{tabular}{|c|c|c|c|}
\hline Year & Active Firms & Defaults & $(\%)$ \\
\hline 1990 & 1,244 & 1 & 0.08 \\
\hline 1991 & 1,681 & 4 & 0.24 \\
\hline 1992 & 2,072 & 12 & 0.58 \\
\hline 1993 & 2,242 & 6 & 0.27 \\
\hline 1994 & 2,322 & 9 & 0.39 \\
\hline 1995 & 2,374 & 11 & 0.46 \\
\hline 1996 & 2,398 & 14 & 0.58 \\
\hline 1997 & 2,471 & 10 & 0.40 \\
\hline 1998 & 2,526 & 19 & 0.75 \\
\hline 1999 & 2,815 & 20 & 0.71 \\
\hline 2000 & 2,912 & 20 & 0.69 \\
\hline 2001 & 2,985 & 41 & 1.37 \\
\hline 2002 & 3,150 & 41 & 1.30 \\
\hline 2003 & 3,434 & 37 & 1.08 \\
\hline 2004 & 3,548 & 34 & 0.96 \\
\hline 2005 & 3,487 & 39 & 1.12 \\
\hline 2006 & 3,378 & 24 & 0.71 \\
\hline 2007 & 3,406 & 26 & 0.76 \\
\hline 2008 & 3,521 & 83 & 2.36 \\
\hline 2009 & 3,700 & 55 & 1.49 \\
\hline 2010 & 3,906 & 42 & 1.08 \\
\hline 2011 & 3,904 & 39 & 1.00 \\
\hline 2012 & 3,705 & 11 & 0.30 \\
\hline
\end{tabular}


Table 2

Characteristics of the Final Sample: Breakdown by Size and Country

This table presents details on the characteristics of our final sample. Panel A shows descriptive statistics for the distribution of firms and firm-months across size classes. \# of firms is the available number of firms for all years for which we are able to calculate monthly values of the Merton measure. \# of firm-months is the number of observations. We provide also the relative fractions of total firms and firm-months that each size class represents. Finally, the column "Total MC" shows the average total market capitalization of each size class during the years of the study. We measure market capitalization in millions of euros. Panel B presents the breakdown of firms and firm-months by country, with corresponding percentages. Start date is the date at which the information on firms of a given country starts to be available; the end date in our sample, December 2012, is the same for all countries.

\begin{tabular}{|c|c|c|c|c|c|c|c|}
\hline \multicolumn{8}{|c|}{ Panel A. Breakdown by Size } \\
\hline Segment & Size & $\#$ of firms & $(\%)$ & \# of firm-months & $(\%)$ & Total MC & $(\%)$ \\
\hline Nano cap & $<10$ mio & 1,419 & 16.81 & 106,570 & 13.22 & 7,401 & 0.11 \\
\hline Micro cap & $<50$ mio & 2,631 & 31.18 & 219,273 & 27.20 & 68,153 & 1.03 \\
\hline Small cap & $<150$ mio & 1,678 & 19.88 & 158,265 & 19.63 & 150,178 & 2.27 \\
\hline Mid cap & $<1$ bio & 1,855 & 21.98 & 205,855 & 25.54 & 735,025 & 11.11 \\
\hline Large cap & $<50$ bio & 839 & 9.94 & 112,526 & 13.96 & $4,239,777$ & 64.07 \\
\hline Mega cap & $\geq 50$ bio & 17 & 0.20 & 3,668 & 0.45 & $1,417,300$ & 21.42 \\
\hline Overall sample & & 8,439 & & 806,157 & & $6,617,834$ & \\
\hline \multicolumn{8}{|c|}{ Panel B. Breakdown by Country } \\
\hline Country & Start date & \# of firms & $(\%)$ & \# of firm-months & $(\%)$ & & \\
\hline Austria & Jan-90 & 112 & 1.33 & 11,676 & 1.45 & & \\
\hline Belgium & Jan-90 & 151 & 1.79 & 17,842 & 2.21 & & \\
\hline Bulgaria & Mar-08 & 130 & 1.54 & 4,009 & 0.50 & & \\
\hline Czech Republic & Mar-98 & 71 & 0.84 & 3,679 & 0.46 & & \\
\hline Denmark & Jan-90 & 195 & 2.31 & 24,151 & 3.00 & & \\
\hline Finland & Jan-90 & 146 & 1.73 & 18,589 & 2.31 & & \\
\hline France & Jan-90 & 1,126 & 13.34 & 111,829 & 13.87 & & \\
\hline Germany & Jan-90 & 1,104 & 13.08 & 112,428 & 13.95 & & \\
\hline Greece & Oct-90 & 315 & 3.73 & 35,558 & 4.41 & & \\
\hline Hungary & Mar-95 & 45 & 0.53 & 3,558 & 0.44 & & \\
\hline Ireland & Jan-90 & 68 & 0.81 & 8,549 & 1.06 & & \\
\hline Italy & Jan-90 & 340 & 4.03 & 37,353 & 4.63 & & \\
\hline Netherlands & Jan-90 & 213 & 2.52 & 28,940 & 3.59 & & \\
\hline Norway & Jan-90 & 290 & 3.44 & 24,632 & 3.06 & & \\
\hline Poland & Mar-95 & 249 & 2.95 & 10,620 & 1.32 & & \\
\hline Portugal & Oct-90 & 94 & 1.11 & 10,002 & 1.24 & & \\
\hline Romania & Mar-02 & 65 & 0.77 & 2,690 & 0.33 & & \\
\hline Serbia & Jan-12 & 47 & 0.56 & 445 & 0.06 & & \\
\hline Spain & Jan-90 & 175 & 2.07 & 22,619 & 2.81 & & \\
\hline Sweden & Jan-90 & 525 & 6.22 & 42,856 & 5.32 & & \\
\hline Switzerland & Jan-90 & 232 & 2.75 & 31,695 & 3.93 & & \\
\hline United Kingdom & Jan-90 & 2,746 & 32.54 & 242,437 & 30.07 & & \\
\hline Overall Sample & & 8,439 & 100.00 & 806,157 & 100.00 & & \\
\hline
\end{tabular}


Table 3

The Merton Measure and Other Firm Characteristics

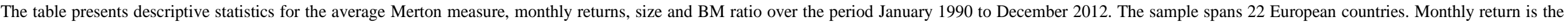

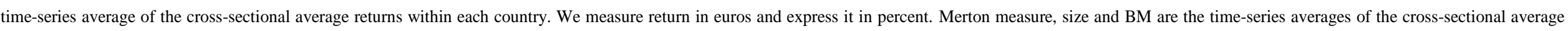
Merton measures, market capitalizations and BM ratios. We express the Merton measure in percentage terms (as it is a probability) and market capitalization in millions of euros.

\begin{tabular}{|c|c|c|c|c|c|c|c|c|c|c|c|c|}
\hline \multirow[b]{2}{*}{ Country } & \multicolumn{3}{|c|}{ Merton measure } & \multicolumn{3}{|c|}{ Monthly Returns } & \multicolumn{3}{|c|}{ Size } & \multicolumn{3}{|c|}{ BM } \\
\hline & Mean & Median & St. Dev. & Mean & Median & St. Dev. & Mean & Median & St. Dev. & Mean & Median & St. Dev. \\
\hline Austria & 4.36 & 3.08 & 3.42 & 0.55 & 0.58 & 5.21 & 541.11 & 313.22 & 389.08 & 0.80 & 0.78 & 0.27 \\
\hline Belgium & 4.70 & 3.96 & 2.88 & 0.63 & 0.90 & 4.16 & 963.20 & 889.92 & 492.07 & 0.84 & 0.81 & 0.18 \\
\hline Bulgaria & 14.29 & 12.99 & 8.46 & -0.64 & 0.28 & 8.18 & 33.02 & 25.84 & 22.61 & 1.74 & 1.83 & 0.35 \\
\hline Czech Republic & 3.31 & 1.25 & 3.87 & 1.28 & 1.33 & 4.38 & 481.78 & 505.48 & 297.82 & 1.72 & 1.47 & 0.68 \\
\hline Denmark & 4.09 & 2.76 & 3.10 & 0.69 & 0.78 & 4.64 & 580.10 & 489.44 & 337.62 & 0.90 & 0.93 & 0.23 \\
\hline Finland & 4.11 & 2.63 & 4.63 & 0.95 & 0.52 & 6.22 & $1,247.22$ & $1,129.59$ & 848.93 & 0.74 & 0.69 & 0.25 \\
\hline France & 5.00 & 4.28 & 2.53 & 0.77 & 0.94 & 4.63 & $1,557.86$ & $1,619.24$ & 576.00 & 0.82 & 0.81 & 0.18 \\
\hline Germany & 4.67 & 3.76 & 3.07 & 0.55 & 0.79 & 3.93 & $1,457.07$ & $1,443.94$ & 431.23 & 0.70 & 0.64 & 0.23 \\
\hline Greece & 6.71 & 4.61 & 5.79 & 1.01 & -0.04 & 10.71 & 197.65 & 176.01 & 137.97 & 1.12 & 0.83 & 0.81 \\
\hline Hungary & 9.14 & 8.76 & 5.24 & 1.62 & 1.15 & 9.39 & 82.84 & 83.77 & 39.55 & 1.33 & 1.33 & 0.48 \\
\hline Ireland & 5.56 & 4.64 & 3.13 & 1.09 & 1.21 & 6.48 & 784.02 & 799.62 & 512.20 & 0.93 & 0.82 & 0.35 \\
\hline Italy & 6.42 & 5.72 & 3.23 & 0.31 & 0.22 & 6.40 & $1,492.03$ & $1,476.92$ & 930.09 & 1.00 & 0.98 & 0.30 \\
\hline Netherlands & 3.42 & 2.91 & 2.21 & 0.58 & 0.86 & 4.93 & $1,832.05$ & $1,866.32$ & 920.79 & 0.75 & 0.72 & 0.21 \\
\hline Norway & 7.37 & 6.85 & 4.23 & 1.11 & 1.43 & 6.83 & 508.20 & 426.60 & 262.46 & 0.89 & 0.86 & 0.32 \\
\hline Poland & 10.27 & 8.57 & 9.51 & 1.31 & 0.69 & 10.82 & 69.94 & 38.80 & 58.53 & 1.27 & 1.04 & 0.76 \\
\hline Portugal & 7.31 & 6.69 & 4.04 & 0.85 & 0.20 & 5.70 & 659.05 & 635.25 & 453.15 & 1.15 & 1.11 & 0.30 \\
\hline Romania & 16.69 & 13.03 & 10.09 & 2.02 & 1.33 & 9.11 & 87.34 & 39.06 & 87.19 & 2.15 & 2.12 & 0.53 \\
\hline Serbia & 12.89 & 13.26 & 3.19 & 0.59 & 0.45 & 5.02 & 17.20 & 16.96 & 2.34 & 3.21 & 3.19 & 0.19 \\
\hline Spain & 4.16 & 3.96 & 2.65 & 0.68 & 0.78 & 5.81 & $2,142.53$ & $1,995.74$ & $1,246.68$ & 0.89 & 0.84 & 0.36 \\
\hline Sweden & 6.64 & 6.21 & 4.13 & 1.02 & 0.91 & 7.05 & $1,084.76$ & 886.09 & 681.68 & 0.77 & 0.74 & 0.28 \\
\hline Switzerland & 3.13 & 2.34 & 2.41 & 0.75 & 0.95 & 4.54 & $2,187.33$ & $2,356.76$ & 961.16 & 0.88 & 0.83 & 0.26 \\
\hline United Kingdom & 4.27 & 3.88 & 2.00 & 0.81 & 1.15 & 5.54 & $1,288.24$ & $1,367.40$ & 559.96 & 0.86 & 0.82 & 0.23 \\
\hline Overall Sample & 5.84 & 4.44 & 5.10 & 0.86 & 0.82 & 6.50 & $1,006.23$ & 765.26 & 896.78 & 0.99 & 0.86 & 0.50 \\
\hline
\end{tabular}


Table 4

\section{Portfolios sorted on the Physical PD}

From January 1990 to December 2012, at the beginning of each month, we sort stocks into quintile portfolios based on their adjusted physical PD in the previous month. We adjust by dividing the physical PDs with the country average for this month. We report results with the Merton measure as a measure of physical PDs. Portfolio 1 is the portfolio with the lowest physical PD and portfolio 5 is the portfolio with the highest physical PD. The portfolios are held for one month and then are rebalanced. Panel A shows the time-series monthly average of the equally and value-weighted portfolio returns and alphas. EMKT is the excess market return, SMB is the return difference between small stocks and big stocks, HML is the return difference between value stocks and growth stocks, and WML is the return difference between winning stocks and losing stocks. The column "High-Low" shows average monthly returns and alphas for portfolios going long the $20 \%$ highest physical PD stocks and short the $20 \%$ lowest stocks. We denominate returns in euros and express them in percentage terms. Panel B shows loadings on the four factors from regressions of the equally and value-weighted excess returns. We calculate the t-statistics in parentheses from Newey-West (1987) standard errors. ** denotes significance at $5 \%$ level and * at $10 \%$ level. Panel C reports PDs, size (in millions of euros), book-to-market ratios (BM), leverage ratios (LR) and return-on-assets (ROA) for each portfolio. SDR betas and IDR alphas are also reported, which will be analyzed in further detail in the following tables.

\begin{tabular}{|c|c|c|c|c|c|c|c|}
\hline Portfolios & High PD 5 & 4 & 3 & 2 & Low PD 1 & High-Low & $t$-stat \\
\hline \multicolumn{8}{|c|}{ Panel A. Portfolio Returns } \\
\hline \multicolumn{8}{|c|}{ Equally-weighted } \\
\hline Return & 0.5776 & 0.5195 & 0.5985 & 0.6502 & 0.6436 & -0.0660 & $(-0.25)$ \\
\hline $\mathrm{CAPM} \alpha$ & 0.2379 & 0.1644 & 0.2569 & 0.3290 & 0.3453 & -0.1075 & $(-0.44)$ \\
\hline 3 -factor $\alpha$ & 0.2269 & 0.1534 & 0.2386 & 0.3130 & 0.3296 & -0.1027 & $(-0.48)$ \\
\hline 4 -factor $\alpha$ & 0.3575 & 0.2195 & 0.2922 & 0.3219 & 0.3197 & 0.0378 & $(0.16)$ \\
\hline \multicolumn{8}{|l|}{ Value-weighted } \\
\hline Return & 0.2062 & 0.4758 & 0.4570 & 0.4597 & 0.6965 & -0.4904 & $(-1.08)$ \\
\hline $\mathrm{CAPM} \alpha$ & -0.1955 & 0.0845 & 0.1014 & 0.1216 & 0.3982 & -0.5936 & $(-1.40)$ \\
\hline 3 -factor $\alpha$ & -0.2704 & 0.0450 & 0.1053 & 0.1518 & 0.4128 & -0.6832 & $(-1.84) *$ \\
\hline 4-factor $\alpha$ & -0.1777 & 0.1973 & 0.1828 & 0.1890 & 0.4675 & -0.6452 & $(-1.63) *$ \\
\hline \multicolumn{8}{|c|}{ Panel B. Four-Factor Regression Coefficients } \\
\hline \multicolumn{8}{|c|}{ Equally-weighted } \\
\hline \multirow[t]{2}{*}{ EMKT } & 0.238 & 0.245 & 0.205 & 0.167 & 0.133 & & \\
\hline & $(3.00) * *$ & $(3.66)^{* *}$ & $(\mathbf{3 . 3 5})^{* * *}$ & $(3.08)^{* * *}$ & $(3.12)^{* *}$ & & \\
\hline \multirow[t]{2}{*}{ SMB } & 1.036 & 0.961 & 0.848 & 0.699 & 0.524 & & \\
\hline & $(6.51)^{* *}$ & $(6.60)^{* * *}$ & $(7.00)^{* * *}$ & $(6.57)^{* * *}$ & $(6.25)^{* *}$ & & \\
\hline \multirow[t]{2}{*}{ HML } & 0.121 & 0.132 & 0.143 & 0.134 & 0.115 & & \\
\hline & $(0.86)$ & $(1.07)$ & $(1.33)$ & $(1.37)$ & $(1.46)$ & & \\
\hline \multirow[t]{2}{*}{ WML } & -0.011 & 0.025 & 0.026 & 0.052 & 0.049 & & \\
\hline & $(-0.14)$ & $(0.35)$ & $(0.41)$ & $(0.86)$ & (1.02) & & \\
\hline
\end{tabular}




\begin{tabular}{|c|c|c|c|c|c|}
\hline \multicolumn{6}{|l|}{ Value-weighted } \\
\hline \multirow[t]{2}{*}{ EMKT } & 0.286 & 0.296 & 0.248 & 0.177 & 0.090 \\
\hline & $(3.28) * *$ & $(3.56)^{* *}$ & $(2.91)^{* *}$ & $(2.52)^{* *}$ & $(2.09)^{* *}$ \\
\hline \multirow[t]{2}{*}{ SMB } & 1.345 & 1.175 & 1.001 & 0.716 & 0.451 \\
\hline & $(6.79) * *$ & $(6.65)^{* *}$ & $(6.69) * *$ & $(\mathbf{5 . 5 5}) * *$ & $(5.24) * *$ \\
\hline \multirow[t]{2}{*}{ HML } & 0.336 & 0.204 & 0.088 & 0.005 & 0.013 \\
\hline & $(1.83)$ & $(1.31)$ & $(0.62)$ & -0.05 & -0.15 \\
\hline \multirow[t]{2}{*}{ WML } & 0.016 & -0.034 & 0.008 & 0.035 & 0.021 \\
\hline & -0.14 & $(-0.35)$ & $(0.09)$ & $(0.43)$ & $(0.31)$ \\
\hline \multicolumn{6}{|c|}{ Panel C. Portfolio Characteristics } \\
\hline Average PD & 22.5600 & 1.7749 & 0.1614 & 0.0096 & 0.0000 \\
\hline Average Size & 286.42 & 530.43 & $1,000.41$ & $1,707.40$ & $2,674.78$ \\
\hline Average BM & 1.4545 & 1.0046 & 0.7706 & 0.6097 & 0.4949 \\
\hline Average LR & 4.0889 & 1.7436 & 1.0925 & 0.7103 & 0.4025 \\
\hline Average ROA & -0.0623 & -0.0045 & 0.0177 & 0.0297 & 0.0369 \\
\hline Average SDR $\beta$ & 0.0590 & 0.1574 & 0.0770 & 0.0327 & 0.0060 \\
\hline Average IDR $\alpha$ & 14.3208 & 0.7767 & -0.0892 & -0.0567 & 0.1510 \\
\hline
\end{tabular}


Table 5

Summary Statistics on VIX

In this table, VIX is the CBOE volatility index and $\Delta_{\mathrm{m}}$ VIX is the monthly change in VIX. Mean, Std, Skew, and Kurt refer to the mean, standard deviation, skewness, and kurtosis, respectively. VSTOXX, VFTSE and VDAX are the EUROSTOXX 50, FTSE 100 and DAX volatility indices, which follow the VIX methodology for the European, UK, and German markets respectively. $\Delta_{\mathrm{m}}$ Eurostoxx50 is the monthly change in EUROSTOXX 50 and $\Delta_{\mathrm{m}}$ MSCIEurope is the monthly change in MSCI Europe. EMKT is the value-weighted excess return on the European market portfolio over the risk-free rate and SMB and HML are the Fama-French factors for Europe. Aggregate PD is the monthly average and Median PD is the monthly median of the Merton measure values of all firms. We calculate the t-statistics from Newey-West (1987) standard errors (up to five lags).

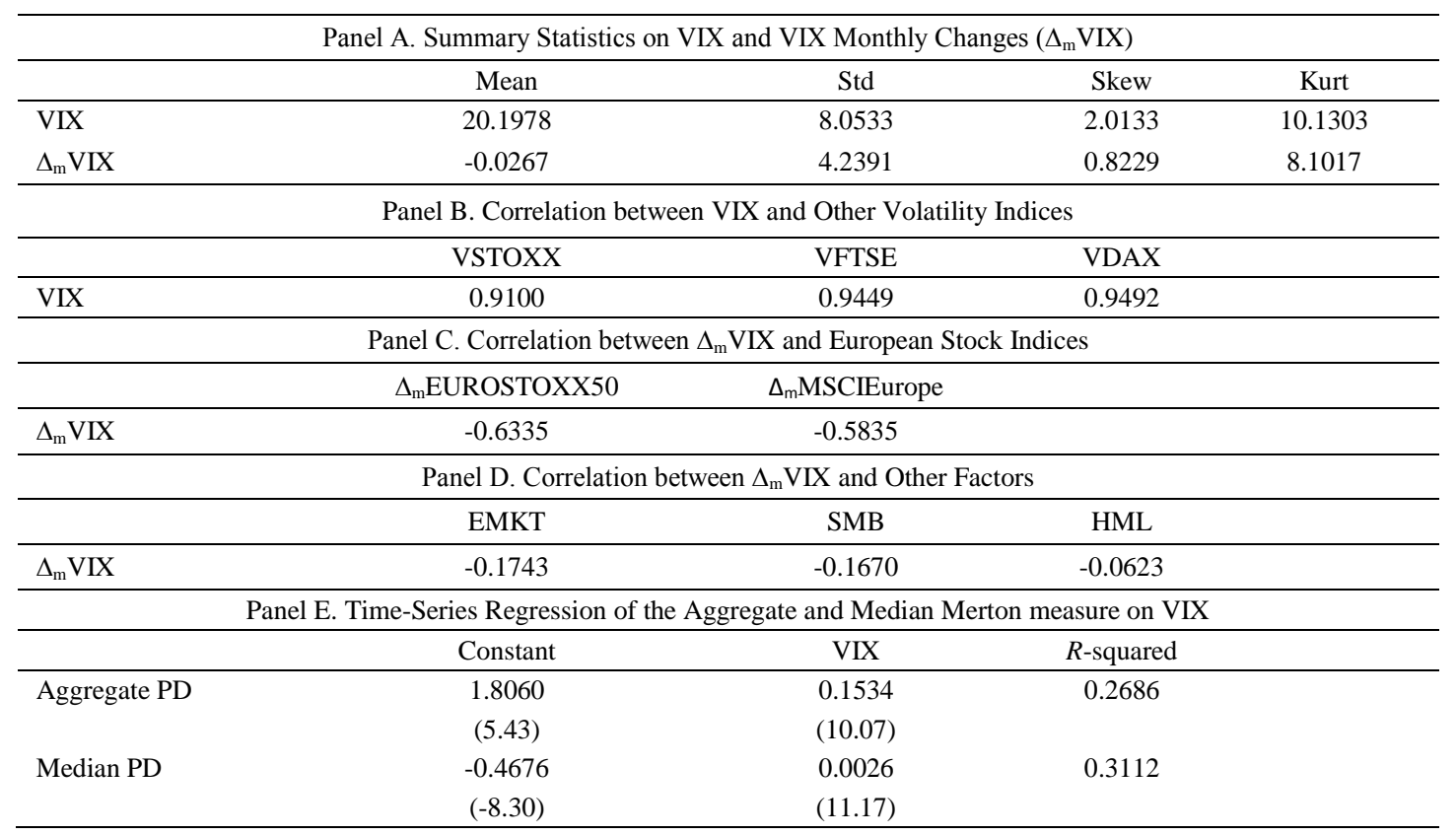


Table 6

Portfolios sorted on the SDR Beta

From January 1992 to December 2012, at the beginning of each month, we sort stocks into quintile portfolios based on their adjusted SDR beta in the previous month. We adjust the SDR betas by demeaning them with the country average for this month. We report results with the VIX SDR beta, which we measure as the coefficient (sensitivity) from 24-months rolling regressions of the PD on VIX. Portfolio 1 is the portfolio with the lowest SDR beta and portfolio 5 is the portfolio with the highest SDR beta. The portfolios are held for one month and then are rebalanced. Panel A shows the time-series monthly average of the equally and value-weighted portfolio returns and alphas. EMKT is the excess market return, SMB is the return difference between small stocks and big stocks, HML is the return difference between value stocks and growth stocks, and WML is the return difference between winning stocks and losing stocks. The column "High-Low" shows average monthly returns and alphas for portfolios going long the $20 \%$ highest SDR beta stocks and short the $20 \%$ lowest stocks. We denominate returns in euros and express them in percentage terms. Panel B shows loadings on the four factors from regressions of the equally and value-weighted excess returns. We calculate the t-statistics in parentheses from Newey-West (1987) standard errors. ** denotes significance at 5\% level and * at 10\% level. Panel C reports PDs, SDR betas, IDR alphas, sizes (in millions of euros), BM, LR and ROA ratios for each portfolio.

\begin{tabular}{|c|c|c|c|c|c|c|c|}
\hline Portfolios & High $\beta 5$ & 4 & 3 & 2 & Low $\beta 1$ & High-Low & $t$-stat \\
\hline \multicolumn{8}{|c|}{ Panel A. Portfolio Returns } \\
\hline \multicolumn{8}{|c|}{ Equally-weighted } \\
\hline Return & 0.8924 & 0.7232 & 0.7175 & 0.7041 & 0.5985 & 0.2939 & $(1.80)^{*}$ \\
\hline CAPM $\alpha$ & 0.5249 & 0.3922 & 0.3889 & 0.3777 & 0.2700 & 0.2549 & $(1.80) *$ \\
\hline 3 -factor $\alpha$ & 0.4577 & 0.3070 & 0.3014 & 0.3317 & 0.1835 & 0.2742 & $(1.89)^{*}$ \\
\hline 4-factor $\alpha$ & 0.4460 & 0.2883 & 0.2750 & 0.2697 & 0.1117 & 0.3343 & $(1.97) * *$ \\
\hline \multicolumn{8}{|c|}{ Value-weighted } \\
\hline Return & 0.8066 & 0.6384 & 0.5877 & 0.5687 & 0.4391 & 0.3675 & $(1.24)$ \\
\hline CAPM $\alpha$ & 0.4162 & 0.3016 & 0.2814 & 0.2720 & 0.0859 & 0.3302 & $(1.14)$ \\
\hline 3 -factor $\alpha$ & 0.3149 & 0.3152 & 0.2153 & 0.2297 & 0.0985 & 0.2164 & $(0.76)$ \\
\hline 4-factor $\alpha$ & 0.4035 & 0.3061 & 0.1989 & 0.1854 & 0.0527 & 0.3508 & $(1.19)$ \\
\hline \multicolumn{8}{|c|}{ Panel B. Four-Factor Regression Coefficients } \\
\hline \multicolumn{8}{|c|}{ Equally-weighted } \\
\hline \multirow[t]{2}{*}{ EMKT } & 0.266 & 0.182 & 0.182 & 0.191 & 0.191 & & \\
\hline & $(3.63)^{* *}$ & $(3.16)^{* *}$ & $(3.72)^{* *}$ & $(3.53)^{* *}$ & $(3.71)^{* * *}$ & & \\
\hline \multirow[t]{2}{*}{ SMB } & 0.979 & 0.715 & 0.767 & 0.726 & 0.771 & & \\
\hline & $(6.28)^{* * *}$ & $(5.66)^{* * *}$ & $(7.02)^{* * *}$ & $(6.67)^{* * *}$ & $(6.30)^{* *}$ & & \\
\hline \multirow[t]{2}{*}{ HML } & 0.148 & 0.197 & $0.204 *$ & 0.118 & 0.216 & & \\
\hline & (1.16) & (1.86) & $(2.05)^{* *}$ & $(1.21)$ & $(2.16)^{* *}$ & & \\
\hline \multirow[t]{2}{*}{ WML } & 0.009 & 0.015 & 0.021 & 0.050 & 0.058 & & \\
\hline & $(0.13)$ & $(0.23)$ & $(0.37)$ & $(0.81)$ & $(0.91)$ & & \\
\hline
\end{tabular}




\begin{tabular}{|c|c|c|c|c|c|}
\hline \multicolumn{6}{|l|}{ Value-weighted } \\
\hline \multirow[t]{2}{*}{ EMKT } & 0.287 & 0.214 & 0.137 & 0.133 & 0.250 \\
\hline & $(3.60)^{* *}$ & $(3.82)^{* *}$ & $(2.54)^{* *}$ & $(2.45)^{* *}$ & $(2.38)^{* *}$ \\
\hline \multirow[t]{2}{*}{ SMB } & 1.060 & 0.763 & 0.652 & 0.683 & 0.770 \\
\hline & $(5.68) * *$ & $(5.04)^{* *}$ & $(6.28)^{* *}$ & $(6.81)^{* *}$ & $(4.55)^{* *}$ \\
\hline \multirow[t]{2}{*}{ HML } & 0.196 & -0.040 & 0.152 & 0.104 & -0.026 \\
\hline & $(1.37)$ & $(-0.36)$ & $(1.47)$ & $(1.08)$ & $(-0.19)$ \\
\hline \multirow[t]{2}{*}{ WML } & -0.071 & 0.007 & 0.013 & 0.036 & 0.037 \\
\hline & $(-0.72)$ & $(0.09)$ & $(0.17)$ & $(0.47)$ & $(0.43)$ \\
\hline \multicolumn{6}{|c|}{ Panel C. Portfolio Characteristics } \\
\hline Average PD & 10.7144 & 1.6788 & 0.5810 & 0.6172 & 8.7870 \\
\hline Average SDR $\beta$ & 0.8881 & 0.0516 & 0.0081 & -0.0025 & -0.6166 \\
\hline Average IDR $\alpha$ & -5.9819 & 0.3573 & 0.3122 & 0.5973 & 18.7048 \\
\hline Average size & 708.81 & $1,691.08$ & $1,957.37$ & $1,964.43$ & $1,044.72$ \\
\hline Average BM & 1.1773 & 0.7985 & 0.6703 & 0.6806 & 1.0280 \\
\hline Average LR & 2.8791 & 1.2538 & 0.8418 & 0.8740 & 2.2675 \\
\hline Average ROA & -0.0251 & 0.0144 & 0.0272 & 0.0256 & -0.0093 \\
\hline
\end{tabular}


Table 7

Portfolios sorted on the IDR Alpha

From January 1992 to December 2012, at the beginning of each month, we sort stocks into quintile portfolios based on their adjusted IDR alpha in the previous month. We adjust the IDR alphas by demeaning them with the country average for this month. We report results with the IDR alpha, which we measure as the constant from 24-months rolling regressions of the PD on VIX. Portfolio 1 is the portfolio with the lowest IDR alpha and portfolio 5 is the portfolio with the highest IDR alpha. The portfolios are held for one month and then are rebalanced. Panel A shows the time-series monthly average of the equally and value-weighted portfolio returns and alphas. EMKT is the excess market return, SMB is the return difference between small stocks and big stocks, HML is the return difference between value stocks and growth stocks, and WML is the return difference between winning stocks and losing stocks. The column "High-Low" shows average monthly returns and alphas for portfolios going long the $20 \%$ highest IDR alpha stocks and short the $20 \%$ lowest stocks. We denominate returns in euros and express them in percentage terms. Panel B shows loadings on the four factors from regressions of the equally and value-weighted excess returns. We calculate the t-statistics in parentheses from Newey-West (1987) standard errors. ** denotes significance at $5 \%$ level and * at $10 \%$ level. Panel C reports PDs, SDR betas, IDR alphas, sizes (in millions of euros), BM, LR and ROA ratios for each portfolio.

\begin{tabular}{|c|c|c|c|c|c|c|c|}
\hline Portfolios & High $\alpha 5$ & 4 & 3 & 2 & Low $\alpha 1$ & High-Low & $t$-stat \\
\hline \multicolumn{8}{|c|}{ Panel A. Portfolio Returns } \\
\hline \multicolumn{8}{|c|}{ Equally-weighted } \\
\hline Return & 0.6686 & 0.5484 & 0.7229 & 0.8372 & 0.8545 & -0.1858 & $(-1.17)$ \\
\hline $\mathrm{CAPM} \alpha$ & 0.3437 & 0.2203 & 0.3940 & 0.5016 & 0.4904 & -0.1467 & $(-1.04)$ \\
\hline 3 -factor $\alpha$ & 0.2648 & 0.1605 & 0.3193 & 0.4281 & 0.4049 & -0.1401 & $(-0.93)$ \\
\hline 4 -factor $\alpha$ & 0.1907 & 0.0888 & 0.2933 & 0.3885 & 0.4263 & -0.2357 & $(-1.32)$ \\
\hline \multicolumn{8}{|l|}{ Value-weighted } \\
\hline Return & 0.4450 & 0.4243 & 0.5613 & 0.6981 & 0.9573 & -0.5124 & $(-1.97)^{* *}$ \\
\hline $\mathrm{CAPM} \alpha$ & 0.0847 & 0.1073 & 0.2678 & 0.3691 & 0.5894 & -0.5046 & $(-2.00)^{* *}$ \\
\hline 3 -factor $\alpha$ & 0.0675 & 0.0905 & 0.2106 & 0.3434 & 0.5682 & -0.5007 & $(-1.86)^{*}$ \\
\hline 4-factor $\alpha$ & 0.0504 & 0.0209 & 0.2197 & 0.3058 & 0.6456 & -0.5952 & $(-1.81)^{*}$ \\
\hline \multicolumn{8}{|c|}{ Panel B. Four-Factor Regression Coefficients } \\
\hline \multicolumn{8}{|c|}{ Equally-weighted } \\
\hline \multirow[t]{2}{*}{ EMKT } & 0.192 & 0.192 & 0.184 & 0.199 & 0.245 & & \\
\hline & $(3.53)^{* *}$ & $(4.10)^{* *}$ & $(3.25)^{* *}$ & $(3.65)^{* *}$ & $(3.35)^{* * *}$ & & \\
\hline \multirow[t]{2}{*}{ SMB } & 0.851 & 0.706 & 0.740 & 0.756 & 0.903 & & \\
\hline & $(6.40)^{* *}$ & $(5.95)^{* *}$ & $(6.73)^{* * *}$ & $(6.60)^{* * *}$ & $(6.18)^{* *}$ & & \\
\hline \multirow[t]{2}{*}{ HML } & 0.198 & 0.154 & 0.174 & 0.175 & 0.182 & & \\
\hline & $(1.93)$ & $(1.68)$ & $(1.69)$ & $(1.69)$ & $(1.42)$ & & \\
\hline \multirow[t]{2}{*}{ WML } & 0.060 & 0.058 & 0.021 & 0.032 & -0.017 & & \\
\hline & $(0.88)$ & (1.02) & $(0.32)$ & $(0.52)$ & $(-0.24)$ & & \\
\hline
\end{tabular}




\begin{tabular}{lrrrrr}
\hline Value-weighted & & & & & \\
EMKT & $\mathbf{0 . 2 6 5}$ & $\mathbf{0 . 1 7 4}$ & $\mathbf{0 . 1 1 0}$ & $\mathbf{0 . 1 9 6}$ & $\mathbf{0 . 2 6 0}$ \\
& $(\mathbf{2 . 5 1})^{* *}$ & $\mathbf{( 3 . 5 1})^{* *}$ & $(\mathbf{2 . 0 1})^{* *}$ & $(\mathbf{3 . 3 3})^{* *}$ & $(\mathbf{3 . 2 7})^{* * *}$ \\
SMB & $\mathbf{0 . 9 7 5}$ & $\mathbf{0 . 6 0 8}$ & $\mathbf{0 . 6 2 2}$ & $\mathbf{0 . 7 3 8}$ & $\mathbf{0 . 9 7 8}$ \\
& $\mathbf{( 5 . 5 0 ) * *}$ & $\mathbf{( 5 . 4 7 ) * *}$ & $\mathbf{( 6 . 3 7 ) * *}$ & $\mathbf{( 5 . 8 5 ) * *}$ & $(\mathbf{5 . 2 3})^{* * *}$ \\
HML & 0.032 & 0.053 & 0.123 & 0.062 & 0.011 \\
& $(0.24)$ & $(0.52)$ & $(1.22)$ & $(0.61)$ & $(0.08)$ \\
WML & 0.014 & 0.056 & -0.007 & 0.030 & -0.062 \\
& $(0.13)$ & $(0.79)$ & $(-0.10)$ & $(0.38)$ & $(-0.61)$ \\
\hline Average PD & 14.1359 & 0.9728 & 0.3755 & 0.9788 & 5.9189 \\
Average SDR $\beta$ & -0.5192 & 0.0186 & 0.0159 & 0.0586 & 0.7511 \\
Average IDR $\alpha$ & 22.5424 & 0.4605 & 0.0017 & -0.3840 & -8.5594 \\
Average size & 685.71 & $1,731.89$ & $2,058.58$ & $1,792.29$ & $1,096.63$ \\
Average BM & 1.2175 & 0.7378 & 0.6510 & 0.7336 & 1.0144 \\
Average LR & 3.3213 & 1.0551 & 0.7350 & 1.0685 & 1.9494 \\
Average ROA & -0.0328 & 0.0195 & 0.0291 & 0.0203 & -0.0036 \\
\hline
\end{tabular}


Table 8

Portfolios sorted on the Physical PD controlled by the IDR alpha

From January 1992 to December 2012, at the beginning of each month, we sort stocks into three portfolios based on their IDR alpha in the previous month. Within each portfolio, we further sort the stocks into three portfolios, based on their past month's PD. We adjust both IDR alphas and PDs by the country average for this month. The sequential two-sort procedure produces 9 portfolios in total. The portfolios are held for one month and then are rebalanced. Panels A shows the time-series monthly average of the value-weighted returns, respectively, for the 9 portfolios as well as average monthly returns and alphas for portfolios going long the 1/3 highest PD stocks and short the $1 / 3$ lowest PD stocks for all three IDR alpha tertiles. We denominate returns in euros and express them in percentage terms. We calculate t-statistics in parentheses from Newey-West (1987) standard errors. ** denotes significance at $5 \%$ level and $*$ at $10 \%$ level. Panel B reports PDs, SDR betas, IDR alphas, sizes (in millions of euros), BM, LR and ROA ratios for each portfolio.

\begin{tabular}{|c|c|c|c|c|c|}
\hline & High PD & Medium PD & Low PD & High-Low & $t$-stat \\
\hline \multicolumn{6}{|c|}{ Panel A. Portfolio Returns } \\
\hline \multicolumn{6}{|l|}{ Return } \\
\hline High & -0.1105 & 0.2944 & 0.6686 & -0.7791 & $(-1.90)^{*}$ \\
\hline Medium & 0.6117 & 0.4217 & 0.6185 & -0.0068 & $(-0.03)$ \\
\hline Low & 0.8658 & 0.8218 & 0.8121 & 0.0537 & $(0.15)$ \\
\hline \multicolumn{6}{|l|}{ CAPM $\alpha$} \\
\hline High & -0.4474 & -0.0749 & 0.3600 & -0.8074 & $(-2.03)^{* * *}$ \\
\hline Medium & 0.2514 & 0.1110 & 0.3369 & -0.0855 & $(-0.34)$ \\
\hline Low & 0.4839 & 0.4371 & 0.4807 & 0.0032 & $(0.01)$ \\
\hline \multicolumn{6}{|l|}{ 3-factor $\alpha$} \\
\hline High & -0.5367 & -0.2476 & 0.3854 & -0.9221 & $(-2.45)^{* *}$ \\
\hline Medium & 0.1781 & 0.0852 & 0.3037 & -0.1256 & $(-0.61)$ \\
\hline Low & 0.3494 & 0.3890 & 0.4682 & -0.1188 & $(-0.43)$ \\
\hline \multicolumn{6}{|l|}{ 4-factor $\alpha$} \\
\hline High & -0.6182 & -0.3495 & 0.3408 & -0.9591 & $(-2.36)^{* * *}$ \\
\hline Medium & 0.2728 & 0.0637 & 0.2931 & -0.0204 & $(-0.08)$ \\
\hline Low & 0.3286 & 0.4680 & 0.4682 & -0.1397 & $(-0.51)$ \\
\hline \multicolumn{6}{|c|}{ Panel B. Portfolio Characteristics } \\
\hline \multicolumn{6}{|c|}{ Average Probability of Default } \\
\hline High $\alpha$ & 24.7076 & 2.0970 & 0.0269 & & \\
\hline Medium $\alpha$ & 1.4230 & 0.0179 & 0.0002 & & \\
\hline Low $\alpha$ & 10.9104 & 0.9728 & 0.1867 & & \\
\hline \multicolumn{6}{|c|}{ Average SDR Beta } \\
\hline High $\alpha$ & -0.6487 & -0.1936 & -0.0710 & & \\
\hline Medium $\alpha$ & 0.0488 & 0.0079 & 0.0017 & & \\
\hline Low $\alpha$ & 1.0079 & 0.2979 & 0.1347 & & \\
\hline \multicolumn{6}{|c|}{ Average IDR Alpha } \\
\hline High $\alpha$ & 30.4494 & 8.2601 & 2.6320 & & \\
\hline Medium $\alpha$ & 0.0790 & -0.0522 & -0.0042 & & \\
\hline Low $\alpha$ & -10.3704 & -3.8171 & -1.8405 & & \\
\hline \multicolumn{6}{|c|}{ Average Size } \\
\hline High $\alpha$ & 304.65 & 695.78 & $2,305.09$ & & \\
\hline Medium $\alpha$ & 869.65 & $1,964.63$ & $3,044.43$ & & \\
\hline Low $\alpha$ & 525.11 & $1,205.18$ & $2,337.58$ & & \\
\hline
\end{tabular}




\begin{tabular}{lccc}
\hline Average Book-to-Market & & \\
High $\alpha$ & 1.4969 & 0.9854 & 0.6060 \\
Medium $\alpha$ & 0.8975 & 0.6193 & 0.4949 \\
Low $\alpha$ & 1.2925 & 0.8516 & 0.5959 \\
\hline Average Leverage Ratio & & & \\
High $\alpha$ & 4.7897 & 1.8378 & 0.7441 \\
Medium $\alpha$ & 1.3513 & 0.6996 & 0.3793 \\
Low $\alpha$ & 2.8937 & 1.3207 & 0.6827 \\
\hline Average Return-on-Assets & & & \\
High $\alpha$ & -0.0606 & -0.0063 & 0.0274 \\
Medium $\alpha$ & 0.0104 & 0.0318 & 0.0392 \\
Low $\alpha$ & -0.0303 & 0.0138 & 0.0315 \\
\hline
\end{tabular}


Panel A

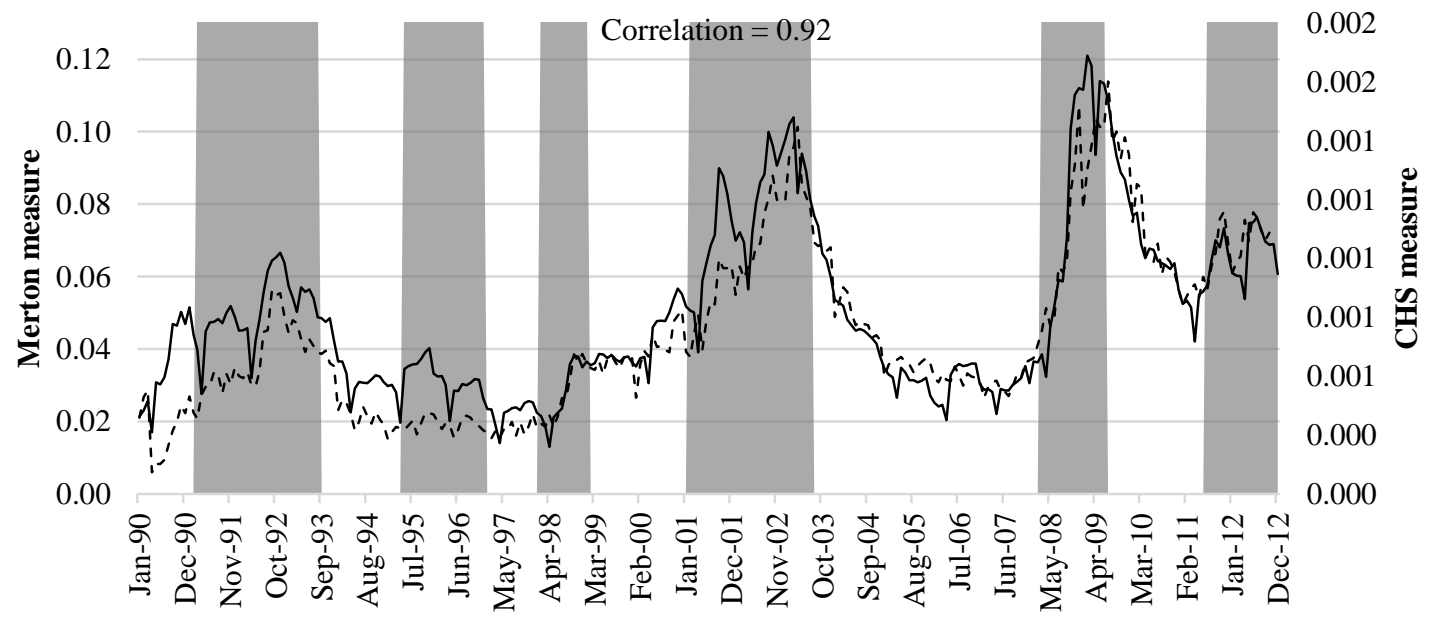

— Merton measure -..- CHS Measure

Panel B

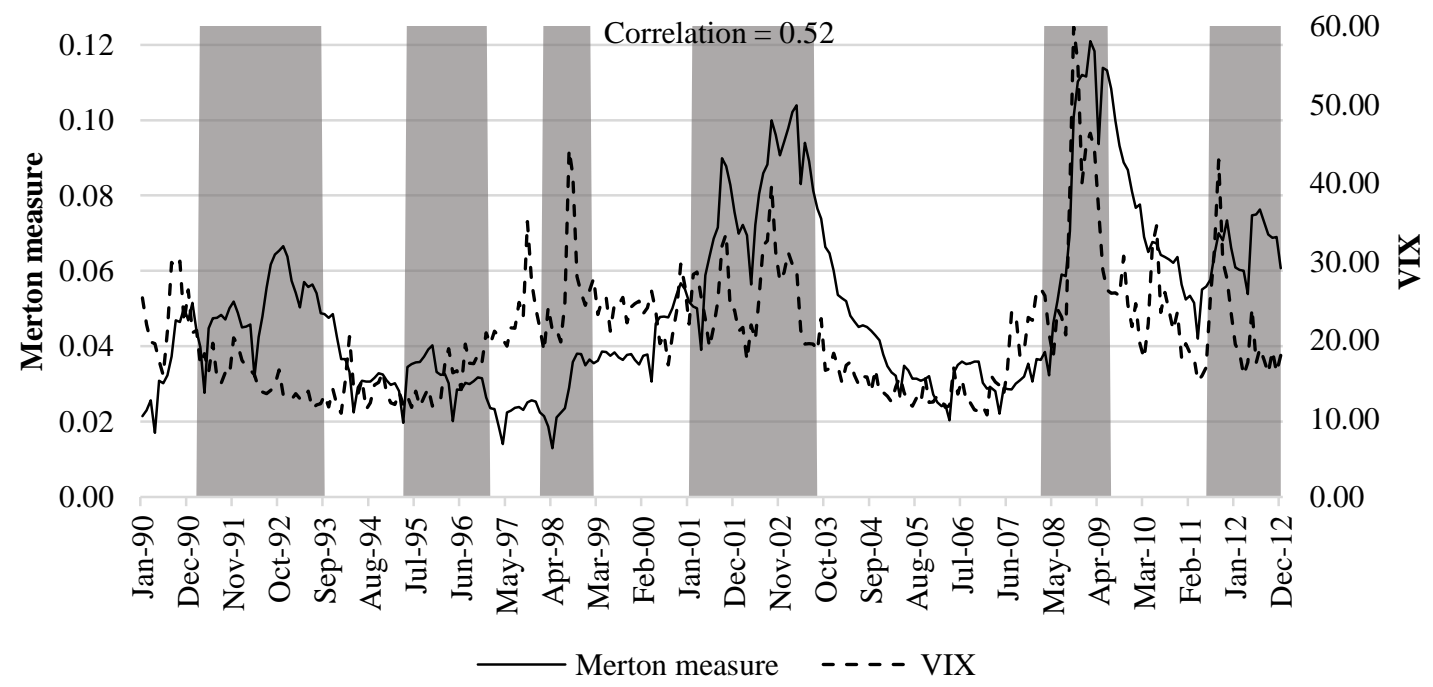

Figure 1. Merton measure, Campbell, Hilscher and Szilagyi Measure and Volatility Index. The figure plots the monthly aggregate Merton (left scale) and CHS (right scale) measures for firms in the overall sample (Panel A) and the monthly aggregate Merton measure (left scale) and monthly VIX (right scale) values (Panel B). We define the aggregate Merton and CHS measures as simple averages of the values of all firms. The Merton measure is the PD estimated following Vassalou and Xing (2002), which we calculate from the Merton's model. The CHS measure is the one used in Campbell, Hilscher and Szilagyi (2008), which we calculate from a dynamic logit model using historical defaults. VIX is available daily from the CBOE and represents a measure of expected stock market volatility. The columns denote recession periods in the Euro area, as indicated by OECD. 


\section{Appendix A. The Merton Measure}

\section{A.I. Calculating the Physical PDs}

Following Vassalou and Xing (2004), we allow only equity and debt in the capital structure of the firm. In Merton's model, equity can be viewed as a call option on firm's assets with a strike price equal to the value of debt. The reason is that equity is a residual claim, i.e. equity holders lay claim to all cash flows left over only after debt holders have been satisfied.

The market value of firm's assets follow a geometric Brownian motion as below:

$$
d V_{A}=\mu V_{A} d t+\sigma_{A} V_{A} d W
$$

where $V_{A}$ is the market value of firm's assets, with an instantaneous drift $\mu$, and instantaneous volatility $\sigma_{A} . W$ is a standard Wiener process.

The market value of firm's equity is given by the Black and Scholes (1973) formula for call options:

$$
\begin{aligned}
& V_{E}=V_{A} N\left(d_{1}\right)-X e^{-r T} N\left(d_{2}\right), \\
& d_{1}=\frac{\ln \left(\frac{V_{A}}{X}\right)+\left(r+\frac{\sigma_{A}^{2}}{2}\right) T}{\sigma_{A} \sqrt{T}}, d_{2}=d_{1}-\sigma_{A} \sqrt{T},
\end{aligned}
$$

where $V_{E}$ is the market value of firm's equity, $X$ is the book value of debt that has a maturity equal to $T, r$ is the risk-free rate, and $N$ is the cumulative density function of the standard normal distribution.

First, we calculate the volatility of equity $\sigma_{E}$ from daily data of the past 12 months and use it as the initial value for the estimation of $\sigma_{A}$. Then, from (2) and (3), we compute $V_{A}$ for each 
trading day of the past 12 months using $V_{E}$ of that day and $X .{ }^{19}$ From the daily values of $V_{A}$ we calculate $\sigma_{A}$ for the next iteration. We repeat this process until the values of $\sigma_{A}$ from two consecutive observations converge. Once we obtain a converged value of $\sigma_{A}$, we use it to find $V_{A}$ from (2) and (3). We repeat the process at the end of every month and obtain monthly values for $\sigma_{A}$. We use the 1-year T-bill rate at the end of the month as the risk-free rate. Once we obtain daily values of $V_{A}$, we compute the drift $\mu$ as the mean of the change in $\ln V_{A}$. Finally, using the normal distribution implied by Merton, we can show that the theoretical PD at time $t$ is given by the following formula:

$$
P D_{t}=N(- \text { Distance to Default } t)=N\left(-\frac{\ln \left(\frac{V_{A, t}}{x_{t}}\right)+\left(\mu-\frac{\sigma_{A}^{2}}{2}\right) T}{\sigma_{A} \sqrt{T}},\right.
$$

where we refer to $P D_{t}$ as the Merton measure.

\section{A.II. Evaluating the Performance of the Merton Measure}

In order to evaluate the performance of the Merton measure, we employ two widely used measures, the Hosmer and Lemeshow grouping and the area under the Receiver Operating Characteristic (ROC) curve.

First, based on the Hosmer and Lemeshow method, each month we rank the estimated PDs and divide them into deciles. Out of the ten groups created (each one containing $1 / 10$ of the firms

\footnotetext{
${ }^{19}$ As Vassalou and Xing (2004) and KMV do, we use current liabilities (WC03101) plus half the longterm debt (WC03251) to calculate the book value of debt $X$. Also, to account for reporting delays that may influence data availability, we use the book value of debt at the fiscal year end, only after 4 months have passed from the fiscal year end.
} 
in that month), the first group has the smallest average estimated PD and the last the largest. Next, we aggregate the number of defaulted firms in each decile for each month over the sample period and calculate the corresponding percentages of the defaulted firms in each decile. The percentage of defaulted firms in the last decile is 58.72. When we look at the last three deciles, this percentage becomes 79.87. This provides us initial evidence that the Merton measure captures important default-related information.

Second, we construct the area under the ROC curve (AUC) from the estimated PDs versus the actual status of the firms in each month for all possible cut-off probability values. Specifically, the curve plots the ratio of correctly classified defaulted firms to actual defaulted firms (sensitivity) and the ratio of wrongly classified healthy firms to actual healthy firms (1 specificity) for all possible cut-offs. The AUC ranges from zero to one. A model with an AUC close to 0.5 is considered a random model with no discriminatory power. An AUC of 0.7 to 0.8 represents good discriminatory power, an AUC of 0.8 to 0.9 very good discriminatory power and an AUC over 0.9 is exceptional and extremely unusual. The AUC that we obtain is equal to 0.8212. This result further supports our belief that the Merton measure is indeed a good default predictor.

As a supplementary and final test, we follow Vassalou and Xing (2004) and compare the PDs of the defaulted firms (treatment group) with the PDs of a group of non-defaulted firms (control group). For each defaulted firm, we choose a healthy firm of similar size (market capitalization) and same industry (4-digit ICB code). We try to match the size of defaulted and healthy firms on the exact month or year of delisting due to default whenever possible. Figure A1 shows the 
average PDs of both groups up to 160 months before delisting. It is apparent that the PDs of both groups move closely together up to four years (48 months) before delisting. In the beginning of the fourth year before delisting though, the average PD of the treatment group goes up sharply, whereas the average PD of the control group does not follow this extreme behavior. Its moderate upward movement can be attributed to general worsening economic conditions in times of many defaults that move upward all PDs in the economy. The average PD at $t=0$ is 0.14 for healthy firms and 0.34 for defaulted firms (around 1.5 times higher). This final test provides additional support that the Merton measure captures default risk successfully. 


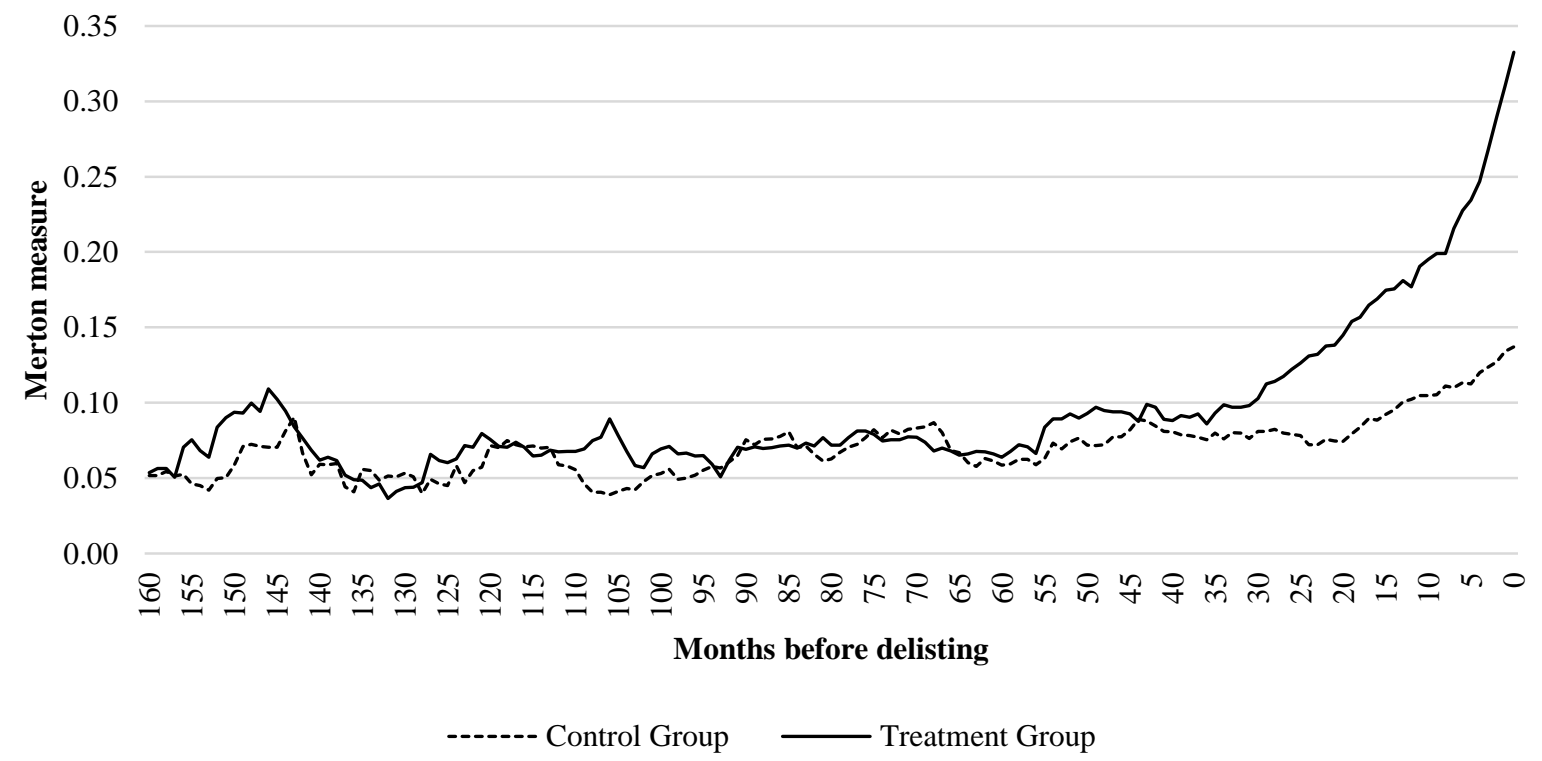

Figure A1. Average Merton Measure of treatment group (defaulted firms) and control group (healthy firms). We choose firms in the control group that have similar size (market capitalization) and same four-digit industry code as those in the treatment group. Specifically for size, we select firms that have similar size with their defaulted counterparts immediately before they delist. We also match the month or year of delisting whenever possible. 


\section{Appendix B. Calculation of the CHS Measure}

Following Campbell, Hilscher and Szilagyi (2008), we use eight variables to calculate the CHS measure (all converted in euros). NIMTA is the ratio of net income (WC07250) to the marketadjusted version of total assets, where the latter is the sum of the market value of equity and the book value of liabilities (WC03351); TLMTA is the ratio of total liabilities to the marketadjusted version of total assets; EXRET is the monthly log excess return relative to the MSCI index of the country that is the firm's main market; ${ }^{20}$ SIGMA is the standard deviation of the daily returns over the previous year; RSIZE is the log ratio of firm's market value to the total market value of firms in the same country and month; CASHMTA is the ratio of cash and shortterm investments (WC02001) to the market-adjusted version of total assets; MB is the market-tobook ratio; and PRICE is the log price truncated at the first and third quartiles of the pooled price distribution. We truncate all other variables at the first and ninety-ninth percentile of their pooled distributions. We lag all accounting data by at least 4 months and market data by 1 month, to ensure their availability at the time of default prediction. To avoid excluding firms shortly before they default, we use data for up to 12 months if more recent data are unavailable.

Table B1 presents summary statistics of these variables. A comparison of Panels B and C reveals the differences of defaulted observations. They have lower profitability, higher leverage, lower stock excess returns, higher stock volatility, lower MB ratios and lower prices compared to healthy observations. They are also smaller. Finally, contrary to the findings of Campbell et al.

\footnotetext{
${ }^{20}$ Robustness checks using other indices yield same results.
} 
(2008) for the US, but in accordance with Aretz, Florackis and Kostakis (2013) for Europe, they hold on average more cash.

Concerning the applied estimation method, we assume that the marginal probability of default over the next period follows a logistic distribution and is given by:

$$
P D\left(Y_{i, t}=1 \mid x_{i, t-1}\right)=\frac{1}{1+\exp \left(-\alpha-\beta x_{i, t-1}\right)},
$$

where $Y_{i, t}$ is an indicator that equals one if the firm defaults in period $t$ and zero otherwise, i.e. if the firm disappears from the sample for some reason other than default, such as delisting due to a merger; and $\beta x_{i, t-1}$ is a function of firm-specific characteristics that includes a vector of predictor variables $x_{i, t-1}$ known at the end of the previous period. Finally, to capture crosscountry differences, we follow two different methods: (i) we estimate separate models for each country; (ii) we introduce country fixed effects and estimate only one model.

Table B2 reports the regression results only under method (ii) due to space limitations. The coefficients confirm the findings from Table $\mathrm{C} 1$. The $\mathrm{CHS}$ measure is negatively related to profitability (NIMTA), excess return (EXRET), size (RSIZE), and PRICE. It is positively related to leverage (TLMTA), volatility (SIGMA), liquidity (CASHMTA) and MB. Most coefficients are significant at a $5 \%$ level, with the exception of CASHMTA and MB. The pseudo- $\mathrm{R}^{2}$ (McFadden's $\mathrm{R}^{2}$ ) is $17.4 \%$, indicating a rather good fit. The pseudo- $\mathrm{R}^{2}$ may look low when compared to $\mathrm{R}^{2}$ values of linear regression models, but such low values are normal in logistic regression. 
Table B1

\section{Summary Statistics for the CHS Measure}

The table reports summary statistics for all of the accounting and market variables used to calculate the CHS measure. NIMTA is the ratio of net income (WC07250) to the market-adjusted version of total assets, where the latter is the sum of the market value of equity and the book value of liabilities (WC03351); TLMTA is the ratio of total liabilities to the market-adjusted version of total assets; EXRET is the monthly $\log$ excess return relative to the MSCI index of the country that is the firm's main market; RSIZE is the log ratio of firm's market value to the total market value of firms in the same country and month; SIGMA is the standard deviation of the daily returns over the previous year; CASHMTA is the ratio of cash and short-term investments (WC02001) to the market-adjusted version of total assets; MB is the market-tobook ratio; and PRICE is the log price truncated at the first and third quartiles of the pooled price distribution. All other variables are truncated at the first and ninety-ninth percentile of their pooled distributions. Panel A describes the distributions of the variables in all observations, Panel B describes the sample of healthy observations, and Panel C describes the defaulted observations.

\begin{tabular}{|c|c|c|c|c|c|c|c|c|}
\hline & NIMTA & TLMTA & EXRET & RSIZE & SIGMA & CASHMTA & MB & PRICE \\
\hline \multicolumn{9}{|c|}{ Panel A. All } \\
\hline Mean & 0.01 & 0.45 & 0.00 & -7.98 & 0.41 & 0.09 & 2.33 & 1.65 \\
\hline Median & 0.03 & 0.44 & -0.01 & -8.04 & 0.36 & 0.06 & 1.63 & 1.62 \\
\hline Std.Dev. & 0.06 & 0.23 & 0.10 & 2.41 & 0.20 & 0.09 & 2.03 & 1.03 \\
\hline Min & -0.16 & 0.07 & -0.19 & -12.01 & 0.15 & 0.00 & 0.40 & 0.39 \\
\hline Max & 0.09 & 0.84 & 0.19 & -3.61 & 0.91 & 0.33 & 8.22 & 2.92 \\
\hline $\mathrm{N}$ & 761,779 & 761,897 & 796,573 & 803,106 & 803,106 & 761,578 & 802,965 & 803,106 \\
\hline \multicolumn{9}{|c|}{ Panel B. Healthy } \\
\hline Mean & 0.01 & 0.45 & 0.00 & -7.98 & 0.41 & 0.09 & 2.33 & 1.65 \\
\hline Median & 0.06 & 0.23 & 0.10 & 2.41 & 0.20 & 0.09 & 2.03 & 1.03 \\
\hline Std.Dev. & 0.03 & 0.44 & -0.01 & -8.04 & 0.36 & 0.06 & 1.63 & 1.62 \\
\hline $\mathrm{N}$ & 761,257 & 761,374 & 795,979 & 802,511 & 802,511 & 761,055 & 802,370 & 802,511 \\
\hline \multicolumn{9}{|c|}{ Panel C. Defaulted } \\
\hline Mean & -0.07 & 0.64 & -0.05 & -10.56 & 0.66 & 0.10 & 1.48 & 0.74 \\
\hline Median & 0.09 & 0.25 & 0.13 & 1.88 & 0.24 & 0.11 & 1.90 & 0.74 \\
\hline Std.Dev. & -0.08 & 0.77 & -0.04 & -11.39 & 0.71 & 0.05 & 0.69 & 0.39 \\
\hline $\mathrm{N}$ & 522 & 523 & 594 & 595 & 595 & 523 & 595 & 595 \\
\hline
\end{tabular}


Table B2

Regression Results for the CHS Measure

The table reports results from the multi-period logit regression of the default indicator on the eight predictor variables. NIMTA is the ratio of net income (WC07250) to the market-adjusted version of total assets, where the latter is the sum of the market value of equity and the book value of liabilities (WC03351); TLMTA is the ratio of total liabilities to the market-adjusted version of total assets; EXRET is the monthly log excess return relative to the MSCI index of the country that is the firm's main market; RSIZE is the log ratio of firm's market value to the total market value of firms in the same country and month; SIGMA is the standard deviation of the daily returns over the previous year; CASHMTA is the ratio of cash and short-term investments (WC02001) to the market-adjusted version of total assets; MB is the market-to-book ratio; and PRICE is the log price truncated at the first and third quartiles of the pooled price distribution. We truncate all other variables at the first and ninety-ninth percentile of their pooled distributions. We lag all accounting data by at least 4 months and market data by 1 month. The model is estimated for January 1990 to December 2012, with yearly observations. Parameter estimates are given first followed by chi-square values in parentheses. Standard errors are cluster-robust to correct for dependence between firm-year observations of the same firm. Numbers significant at the $5 \%$ level are in bold.

\begin{tabular}{lcc}
\hline NIMTA & $\mathbf{- 4 . 4 4 9}$ & $\mathbf{( - 7 . 1 5 )}$ \\
TLMTA & $\mathbf{2 . 9 1 4}$ & $\mathbf{( 1 1 . 3 3 )}$ \\
EXRET & $\mathbf{- 1 . 5 5 0}$ & $\mathbf{( - 3 . 9 1 )}$ \\
RSIZE & $\mathbf{- 0 . 4 5 5}$ & $\mathbf{( - 1 0 . 2 9 )}$ \\
SIGMA & $\mathbf{2 . 3 1 1}$ & $\mathbf{( 9 . 1 9 )}$ \\
CASHMTA & 0.367 & $\mathbf{( 0 . 7 5 )}$ \\
MB & 0.014 & $\mathbf{( 0 . 5 1 )}$ \\
PRICE & $\mathbf{- 0 . 2 5 3}$ & $\mathbf{( - 3 . 3 2 )}$ \\
Constant & $\mathbf{- 1 4 . 1 6 0}$ & $\mathbf{( - 2 7 . 5 4 )}$ \\
Firm-year observations & 755,243 & \\
Firms & 7,980 & \\
Distressed firms & 522 & \\
Country fixed effects & Yes \\
Pseudo R-squared & 0.174 & \\
Log likelihood & -3568.9 & \\
Wald test & 970.0 & \\
\hline
\end{tabular}

